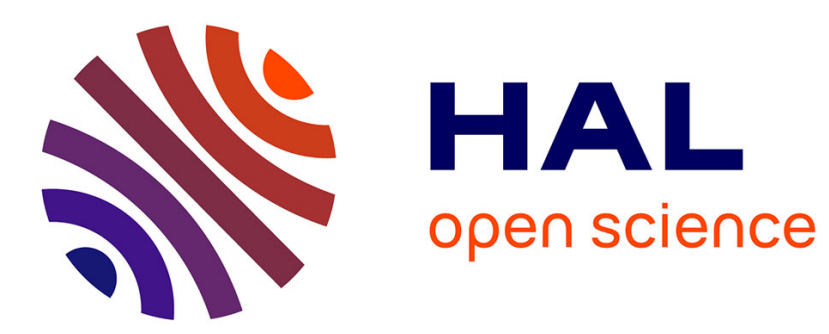

\title{
Monde insulindien et péninsule indochinoise : un panorama de contacts millénaires
}

\author{
Daniel Perret, Danny Wong Tze-Ken
}

\section{To cite this version:}

Daniel Perret, Danny Wong Tze-Ken. Monde insulindien et péninsule indochinoise : un panorama de contacts millénaires. Archipel, 2013, 85 (1), pp.7 - 42. 10.3406/arch.2013.4383 . hal-02565609

\section{HAL Id: hal-02565609 \\ https://hal.science/hal-02565609}

Submitted on 6 May 2020

HAL is a multi-disciplinary open access archive for the deposit and dissemination of scientific research documents, whether they are published or not. The documents may come from teaching and research institutions in France or abroad, or from public or private research centers.
L'archive ouverte pluridisciplinaire HAL, est destinée au dépôt et à la diffusion de documents scientifiques de niveau recherche, publiés ou non, émanant des établissements d'enseignement et de recherche français ou étrangers, des laboratoires publics ou privés. 
Monde insulindien et péninsule indochinoise : un panorama de contacts millénaires

Daniel Perret, Danny Wong Tze-Ken

\section{Citer ce document / Cite this document :}

Perret Daniel, Wong Tze-Ken Danny. Monde insulindien et péninsule indochinoise : un panorama de contacts millénaires. In: Archipel, volume 85, 2013. pp. 7-42;

doi : https://doi.org/10.3406/arch.2013.4383

https://www.persee.fr/doc/arch_0044-8613_2013_num_85_1_4383

Fichier pdf généré le 08/11/2019 


\title{
Résumé
}

Les relations historiques entre différentes régions d'Asie du Sud-Est restent une approche encore largement sous-étudiée, surtout lorsqu'il s'agit d'examiner ces relations sur la longue durée. Malgré des avancées notables depuis une trentaine d'années, l'histoire des relations entre le monde insulindien et la péninsule indochinoise, deux aires qui vues globalement ont emprunté des chemins divergents sur les plans linguistique, religieux et politique notamment, n'échappe pas à ce constat. Afin de stimuler ces recherches, ce panorama diachronique présente, à partir de données les plus récentes, plusieurs jalons couvrant quelque 13000 ans de contacts possibles ou avérés depuis le "phénomène hoabinhien " jusqu'aux principaux phénomènes migratoires observables aujourd'hui. Jusqu'au VIle siècle EC, les données encore très fragmentaires ne permettent pas pour l'instant d'aller au-delà de la mise en lumière d'espaces et de réseaux caractérisés par des traits communs. Ensuite, progressivement la multiplication des événements, toponymes et personnages connus permet de mieux préciser ces relations et d'envisager des approches thématiques multiples allant de la culture matérielle jusqu'à la circulation des idées.

\begin{abstract}
Maritime Southeast Asia and Indochina : A Panorama of Contacts Across Millennia.

Historical relations between regions of Southeast Asia remains a still largely under-studied approach, especially for relations in the longue durée. Although significant progresses have been made during the last thirty years, history of relations between Maritime Southeast Asia and Indochina, two regions that took divergent paths, especially regarding languages, religions and politics, is not an exception to this observation. In order to stimulate these researches, this diachronic panorama, based on most recent data, offers a number of milestones along the 13,000 years of possible or attested contacts, from the "Hoabinhian phenomena" until the main migratory flows visible today. Until the 7th century AD, the still very fragmentary data do not allow to go beyond the observation of spaces and networks sharing a number of common features. Then, the gradual increase in the number of known events, place names and individuals, allows a better insight into these linkages and provides opportunities to conduct multiple thematic approaches, from material culture to circulation of ideas.
\end{abstract}




\title{
Monde insulindien et péninsule indochinoise : un panorama de contacts millénaires*
}

\author{
Daniel Perret \& Danny Wong Tze Ken
}

Parmi les multiples approches possibles de l'histoire de l'Asie du SudEst, il en est une qui semble ne pas avoir encore reçu toute l'attention qu'elle mérite. À notre connaissance, il n'y a en effet pas jusqu'à présent de réflexion générale sur l'histoire des relations entre les différentes régions la constituant. Pourtant, dès 1944, dans son Histoire ancienne des États hindouisés d'Extrême-Orient, Georges Cœdès appliquait le concept de mer Méditerranée à l'ensemble formé par la mer de Chine, le golfe de Siam et la mer de Java. Il imaginait cette mer plus comme un potentiel d'interactions pour les populations côtières que comme une barrière ${ }^{1}$. Plus près de nous, dès la première page de son Carrefour javanais, Denys Lombard insistait sur l'importance des rapports entre l'Archipel, le monde mon-khmer et l'ensemble de la péninsule indochinoise, et un peu plus loin reprenait l'idée de Cœdès sur la mer comme espace d'échanges en suggérant de considérer les étendues maritimes comme des zones de gravité ${ }^{2}$. Quelques années plus tard, il constatait le petit nombre d'études consacrées aux relations entre différentes régions d'Asie du Sud-Est ${ }^{3}$. Dans une toute récente synthèse sur l'histoire de l'Asie du Sud-Est, un chapitre est consacré à cet aspect, mais il est limité à l'histoire des acteurs «non indigènes» présents dans la région ${ }^{4}$.

\footnotetext{
* Ce texte introduit un dossier basé sur une sélection de communications présentées lors du séminaire "Historical Relations between Indochina and the Malay World", qui s'est tenu à Kuala Lumpur les 20-21 octobre 2009, organisé conjointement par la Faculté des Arts et Sciences Sociales de l'University of Malaya et l'École française d'Extrême-Orient à Kuala Lumpur.

1. Les États hindouisés d'Indochine et d'Indonésie, Paris, de Boccard, 3è éd., 1964, réimprimée en $1989: 16$.

2. Le Carrefour javanais. Essai d'histoire globale. I : Les limites de l'occidentalisation, Paris, Éditions de l'École des Hautes Études en Sciences Sociales, 1990 : 13, 15. Voir également Lombard, 1998 et 2007, dans la bibliographie. Afin d'éviter les répétitions, les références mentionnées dans la bibliographie consacrée aux relations entre le monde insulindien et la péninsule indochinoise publiée dans ce dossier, apparaissent uniquement sous une forme abrégée dans cette introduction. Les abréviations utilisées ici sont également détaillées dans la bibliographie.

3. "Networks and Synchronisms in Southeast Asian History", JSEAS, 26(1), 1995: 10-16.

4. M.C. Ricklefs et alii, A New History of Southeast Asia, New York, Palgrave Macmillan, 2010: chapitre 6 "Non-Indigenous Actors Old and New" (p. 116-133).
} 
Le champ n'est toutefois pas complètement vierge d'études détaillées consacrées à l'histoire des relations entre deux ou plusieurs zones de la région. Citons, à titre d'exemples, l'étude de Khin Sok sur le Cambodge et ses voisins entre la fin du XVIII ${ }^{\mathrm{e}}$ siècle et la fin du XIX ${ }^{\mathrm{e}}$ siècle $^{5}$ ou encore le travail de Suwannathat-Pian sur les relations thaïes-malaises entre le XVII siècle et le début du $\mathrm{XX}^{\mathrm{e}}$ siècle $^{6}$. Mais ces recherches restent très peu nombreuses.

Le but de ce dossier est de mettre en lumière cette approche consacrée aux échanges interrégionaux en Asie du Sud-Est à travers le cas des relations sur la longue durée entre le monde insulindien et la péninsule indochinoise, en particulier l'espace représenté aujourd'hui par le Viêt Nam et le Cambodge, deux aires qui, vues globalement, ont emprunté des chemins divergents sur les plans linguistique, religieux et politique. Il n'est bien sûr pas question ici de traiter de tous les aspects de ces échanges multimillénaires.

Cette introduction n'a d'autre prétention que de proposer quelques jalons et d'illustrer les multiples facettes de ces relations réelles ou "imaginées». Elle permet également de situer les contributions de ce dossier dans la longue durée des contacts.

\section{Vestiges d'espaces et de réseaux}

Les indices les plus anciens, quoiqu'encore incertains, de contacts culturels entre le monde insulindien et la péninsule indochinoise remontent à environ 13000 ans, avec ce que les préhistoriens s'accordent à nommer le phénomène hoabinhien, qui se traduit par l'existence d'une industrie lithique, précisément des outils sur galets, que l'on retrouve en péninsule indochinoise, en péninsule malaise et à Sumatra. Une industrie qui pourrait d'une part, être l'héritière de pratiques en usage chez les chasseurs-cueilleurs des mêmes régions, il y a quelque 35000 ans, et qui d'autre part, va perdurer jusqu'au développement de l'agriculture ${ }^{7}$. Les aborigènes Semang Negritos de la péninsule malaise, locuteurs de langues austro-asiatiques, sont probablement des descendants de ces populations adeptes de l'outillage sur galet $^{8}$.

5. Le Cambodge entre le Siam et le Viêtnam : de 1775 à 1860, Paris, École française d'Extrême-Orient, 1991.

6. Thai-Malay Relations: Traditional Intra-Regional Relations from the Seventeenth to the Early Twentieth Centuries, Singapore/New York, Oxford University Press, 1988.

7. Hubert Forestier, Truman Simanjuntak, Florent Détroit, Valéry Zeitoun, "Unité et diversité préhistorique entre Java et Sumatra", Archipel, 80, 2010 : 23 (19-44).

8. $\mathrm{P}$. Bellwood considère que le facteur linguistique ne prouve pas que ces aborigènes soient originaires de la région qui est aujourd'hui le Cambodge, ajoutant que leur présence en péninsule malaise semble plutôt liée à l'expansion de la culture dite Ban Kao, centrée il y a quelque 2000 ans sur la province thaïe actuelle de Kanchanaburi (Bellwood, "Southeast Asia before History", in N. Tarling (ed.), The Cambridge History of Southeast Asia, Cambridge, Cambridge University Press, I, 1992: 100-1 (55-136)). 
Selon le modèle archéo-linguistique le plus largement accepté aujourd'hui, des populations de l'ouest de Bornéo appartenant à la famille linguistique chamique s'installent sur les côtes méridionales du Viêt Nam entre le début du premier millénaire avant notre ère et le tout début de notre ère ${ }^{9}$. Les communautés maritimes dites Sa Huynh seraient le fruit de la rencontre entre les populations néolithiques locales et ces nouveaux arrivants ${ }^{10}$. Cette culture Sa Huynh emprunte son nom au site archéologique situé dans le sud du Viêt Nam, découvert au tout début du XX ${ }^{\mathrm{e}}$ siècle. Elle a été identifiée également dans le delta du Mékong, au sud de la Thaillande, à Bornéo (grotte de Niah à Sarawak), ainsi qu'aux Philippines ${ }^{11}$. Se dessine ainsi un réseau à travers les deux zones qui nous intéressent ici, réseau qui traduit probablement l'existence de contacts culturels et commerciaux à 1'époque ${ }^{12}$. Au Viêt Nam, ces sites de tradition Sa Huynh pourraient représenter les premières implantations cam et cette culture semblerait aussi à l'origine de l'introduction des techniques de travail du fer en Asie du SudEst insulaire ${ }^{13}$. Rappelons ici que le plus ancien document en langue austronésienne connu à ce jour est l'inscription cam de Dong Yen Chau, datable du IVe siècle EC ${ }^{14}$.

Un autre métal a joué un rôle important dans l'histoire des contacts entre la péninsule indochinoise et le monde insulindien, il s'agit du bronze. Le

9. P. Bellwood, 1992: 114-115; P. Bellwood, Prehistory of the Indo-Malaysian Archipelago, Honolulu, Univ. of Hawai'i Press, 1997 (1 ère éd., 1985): 120-121; P. Bellwood, "The Origins and Dispersals of Agricultural Communities in Southeast Asia", in I. Glover \& P. Bellwood (eds.), Southeast Asia: From Prehistory to History, London/New York, RoutledgeCurzon, 2004: 28-29 (21-40).

10. Bellwood, 1992: 130 (55-136); Bellwood, 1997: 271-2; Charles Higham, Early Cultures of Mainland Southeast Asia, Bangkok, River Books, 2002: 179-183.

11. Pour un point récent sur cette question aux Philippines, voir E.A. Bacus, "The Archaeology of the Philippine Archipelago", in I. Glover \& P. Bellwood (eds.), Southeast Asia: From Prehistory to History, London/New York, RoutledgeCurzon, 2004: 263-266 (257-281).

12. P. Bellwood, 2004: 38 (21-40). Pour une étude de cas très récente, voir B. Bellina, G. Epinal, A. Favereau, "Caractérisation préliminaire des poteries marqueurs d'échanges en mer de Chine méridionale à la fin de la préhistoire", Archipel, 84, 2012 : 7-33.

13. Pour des éléments récents sur ces questions, voir B. Bellina, E.A. Bacus, T.O. Pryce \& J.W. Christie (eds.), 50 Years of Archaeology in Southeast Asia. Essays in Honour of Ian Glover, Bangkok, River Books, 2010; P. Bellwood, "Southeast Asia before History", in N. Tarling (ed.), The Cambridge History of Southeast Asia, Cambridge, Cambridge University Press, vol. I(1), 1999: 130-1 (55-136); C. Higham, "Mainland Southeast Asia: From the Neolithic to the Iron Age", in I. Glover \& P. Bellwood (eds.), Southeast Asia: From Prehistory to History, London/New York, RoutledgeCurzon, 2004: 60 (41-67); W.A. Southworth, "The Coastal States of Champa", in I. Glover \& P. Bellwood (eds.), Southeast Asia: From Prehistory to History, London/New York, RoutledgeCurzon, 2004: 212-213 (209-233).

14. G. Coedès, Les États hindouisés d'Indochine et d'Indonésie, Paris, de Boccard, 1964 (1 ère ed. 1948) : 96. Cette inscription est translittérée et traduite en anglais dans Thurgood (1999:3). 
développement du travail du bronze en Asie du Sud-Est a très récemment fait l'objet d'une importante révision, étant maintenant situé vers $1000 \mathrm{AE}^{15}$. L'industrie du bronze la plus connue est celle de Dong Son, qui tire son nom du site du même nom, dans la province de Thanh Hoa au sud d'Hanoi, fouillé initialement dans les années 1920 et 1930. La réputation de cette industrie est surtout liée à sa production de tambours dont la forme particulière a peut-être vu le jour vers 600 AE. Un type, probablement fabriqué uniquement au Viêt Nam, nous concerne tout particulièrement ici, c'est le type dit Heger I, puisqu' on en retrouve de nombreux exemplaires non seulement au Viêt Nam, mais aussi au Cambodge, en Thaïlande, ainsi que dans l'Archipel ${ }^{16}$. Fabriqués au moins jusqu'au III ${ }^{\mathrm{e}}$ siècle EC, ils sont parvenus jusque dans le Grand Est indonésien, en particulier l'archipel de Kai près de la Nouvelle-Guinée. Bellwood semble suggérer que de par leur position géographique par rapport à la zone de production, des Austronésiens appartenant à la famille linguistique chamique auraient joué un rôle important dans leur diffusion 17 .

D'autres traits culturels ont pu suivre la même voie. C'est le cas de la charpente à faitière tendue dont la silhouette est représentée sur des tambours de Dong Son (ainsi que du Yunnan) et que l'on retrouve justement en usage jusqu'à l'île de Sangeang au nord-est de Sumbawa ${ }^{18}$. On observe par ailleurs à la même époque des coutumes funéraires similaires sur la côte du centre du Viêt Nam (site de Go Cam), à Java Ouest, ainsi qu'au nord de Bali ${ }^{19}$.

C'est aussi durant les premiers siècles de l'ère chrétienne qu'a pu se produire ce qui serait la première «conquête» connue concernant les deux zones qui nous retiennent ici. Une source chinoise mentionne en effet que le royaume du Funan, centré sur le delta du Mékong, prend le contrôle d'une partie de la péninsule malaise, un événement qui serait à placer au III $^{\mathrm{e}}$ siècle $\mathrm{EC}^{20}$. Le Funan attire les navires marchands de l'Archipel livrant dans ses ports des denrées destinées au marché chinois, en particulier des produits

15. C. Higham, T. Higham, A. Kijngam, "Cutting a Gordian Knot: The Bronze Age of Southeast Asia, Origins, Timing and Impact", Antiquity, 85, 2011: 583-598; C. Higham, T. Higham, R. Ciarla, K. Douka, A. Kijngam, F. Rispoli, "The Origins of the Bronze Age of Southeast Asia", Journal of World Prehistory, 24(4), 2011: 227-274.

16. Selon un décompte effectué dans les années 1980, on arrive à plus de 200, dont un quart en Asie du Sud-Est insulaire (Bellwood, 1999: 122).

17. Bellwood, 1997: 272; Bellwood, 2004: 37.

18. Dumarçay, 1981, 1998.

19. Manguin, 2010: 178-9.

20. G. Cœdès, 1964: 82. M. Vickery ("Funan Reviewed: Deconstructing the Ancients", BEFEO, 90-91, 2003: 124-125), puis E. Bourdonneau ("Réhabiliter le Funan : Óc Eo ou la première Angkor", BEFEO, 94, $2007: 131$ ) ont repris cette question récemment. 
forestiers et les épices des Moluques ${ }^{21}$. Dès le début du $\mathrm{III}^{\mathrm{e}}$ siècle EC, les sources chinoises signalent une autre entité politique côtière, le Linyi, dans la moitié nord du Viêt Nam, qui entretient très probablement des contacts commerciaux avec le monde insulindien ${ }^{22}$.

D'autres indices de réseaux apparaissent au $\mathrm{V}^{\mathrm{e}}$ siècle, marqués par la présence de statues de Viṣnu «mitrés» dans le delta du Mékong, à la fois au Cambodge et au Viêt Nam, en Thaïlande péninsulaire et dans l'Archipel, précisément sur les sites de Kota Kapur sur l'île de Bangka, de Cibuaya à Java Ouest, ainsi qu'à Bali ${ }^{23}$. P.-Y. Manguin associe ces statues particulières à un réseau maritime de marchands adeptes du courant Vaiṣnava reliant plusieurs ports d'Asie du Sud-Est. Dans l'Archipel, le vestige le plus récent de ce réseau serait le site de Kota Kapur daté de la fin du VII ${ }^{\mathrm{e}}$ siècle $^{24}$.

À la même époque, le bouddhisme manifeste également une expression commune sur les routes commerciales maritimes de la région, notamment à travers la statuaire et les inscriptions en sanskrit ${ }^{25}$. Ces témoignages artistiques et épigraphiques, auxquels il faut probablement ajouter certaines techniques architecturales communes, sont complétés depuis une dizaine d'années par du mobilier issu de fouilles archéologiques. En effet, des assemblages archéologiques similaires (poteries, objets en étain, perles, etc...) ont été mis au jour à Oc Eo dans le delta du Mékong, en péninsule malaise (Pontian, Kuala Selinsing), à Sumatra (sites de Air Sugihan/Banyuasin près de l'embouchure de la rivière Musi à Sumatra Sud), à Java Ouest (Batujaya), et enfin à Java Central (Pekalongan) pour un sceau d'influence indienne ou hellénistique similaire à des trouvailles d'Oc Eo ${ }^{26}$.

21. K.R. Hall, "Economic History of Early Southeast Asia", in N. Tarling (ed.), The Cambridge History of Southeast Asia. I: From Early Times to c. 1500, Cambridge, Cambridge Univ. Press, 1999 (1ère éd., 1992): 194 (183-275); Manguin, "The Archaeology of Fu Nan in the Mekong River Delta: the Oc Eo Culture of Viet Nam”, in N. Tingley (ed.), Arts of Ancient Viet Nam: From River Plain to Open Sea, Houston, Asia Society, The Museum of Fine Arts, New Haven/London, Yale Univ. Press, 2009: 111 (103-118).

22. Pour une synthèse sur le Linyi, voir W.A. Southworth, "The Coastal States of Champa", in I. Glover \& P. Bellwood (eds.), Southeast Asia: From Prehistory to History, London/New York, RoutledgeCurzon, 2004: 216-222 (209-233). Sur la question de la localisation de Linyi, voir M. Vickery, "Champa Revised", in Trần Kỳ Phương \& B.M. Lockhart (eds.), The Cham of Vietnam: History, Society and Art, Singapore, National Univ. Press, 2011: 363-420 (en particulier pp. 372-378).

23. Les auteurs remercient Arlo Griffiths d'avoir porté à leur attention cette découverte récente à Bali, ainsi que pour ses suggestions ayant permis d'améliorer une version antérieure de ce texte.

24. Dalsheimer \& Manguin, 1998.

25. Manguin 2010: 172-174.

26. Manguin, 2002, 2004; P.Y. Manguin \& A. Indrajaya, "The Archaeology of Batujaya (West-Java, Indonesia): An interim Report", in E.A. Bacus, I.C. Glover \& V.C. Pigott (eds.), Uncovering Southeast Asia's Past. Selected Papers from the $10^{\text {th }}$ International Conference of 
Au moins une figure mythique relie les deux ensembles pour cette époque ancienne. Elle apparaît dans une tradition encore vivante dans les années 1970 à Java. Il s'agit d'un certain Aji Saka, considéré comme l'acteur principal de l'indianisation de l'île, acteur que cette tradition fait venir du Campā ${ }^{27}$. Le Campā lui-même apparaît comme une entité indépendante au $\mathrm{V}^{\mathrm{e}}$ siècle, entité dont les ports servent déjà d'escale pour les navires de 1'Archipel se rendant en Chine ${ }^{28}$. Une source chinoise du VII ${ }^{\mathrm{e}}$ siècle suggère ainsi que Cua Dai Chiem (province de Quang Nam) et l'archipel de Cu Lao Cham, ont participé activement à ces échanges à l'époque ${ }^{29}$. L'archéologie confirme d'ailleurs cette situation pour des sites en amont sur la rivière Thu Bon, tels que Tra Kieu, Hau Xa et Go Cam ${ }^{30}$.

En ce qui concerne cette période qui débute avec l'horizon hoabinhien et s'achève au VII ${ }^{\mathrm{e}}$ siècle de notre ère, les relations entre le monde insulindien et la péninsule indochinoise sont aujourd'hui perçues essentiellement à travers la mise en lumière d'espaces et de réseaux caractérisés par des traits communs sur le plan de la culture matérielle et de la vie religieuse, et secondairement à travers des sources chinoises à partir des premiers siècles de notre ère. Si elle peut être confirmée un jour, la prise de contrôle d'une partie de la péninsule malaise par le Funan au III siècle EC constituerait, au cours de cette longue période, l'unique événement dont la trace soit parvenue jusqu'à nous à propos des relations qui nous intéressent ici.

\section{Des réseaux qui s'affirment et une histoire événementielle fragmentaire}

Les contours de ces relations commencent à se préciser durant ce VII ${ }^{\mathrm{e}}$ siècle avec l'apparition de noms d'acteurs, de toponymes et d'événements liés à ces relations. C'est tout d'abord la question de l'origine de la dynastie Śailendra à Java, dont les premiers dirigeants ont pu être politiquement actifs

the European Association of Southeast Asian Archaeologists, Singapore, NUS Press, 2006: 245-257; P.Y. Manguin \& A. Indrajaya, "The Batujaya Site: New Evidence of Early Indian Influence in West Java", in P.-Y. Manguin, A. Mani \& G. Wade (eds.), Early Interactions between South and Southeast Asia, Singapore, ISEAS, New Delhi, Manohar, 2011: 129; A. Indrajaya, "The Pre-Srivijaya Period on the Eastern Coast of Sumatra: Preliminary Research at the Air Sugihan Site", in M.L. Tjoa-Bonatz, A. Reinecke \& D. Bonatz (eds.), Connecting Empires and States. Selected Papers from the $13^{\text {th }}$ International Conference of the European Association of Southeast Asian Archaeologists, vol. 2, Singapore, NUS Press, 2012: 32-42.

27. Lombard, 1987: 312.

28. K.R. Hall, A History of Early Southeast Asia. Maritime Trade and Societal Development, 100-1500, Lanhan, etc., Rowman \& Littlefield Pub., 2011: 73, 75.

29. Voir G. Wade dans ce volume.

30. W.A. Southworth, 2004: 221 (209-233). W. Southworth \& R. Prior, "History and Archaeology at Trà Kiệu", in B. Bellina, E.A. Bacus, T.O. Pryce \& J.W. Christie (eds.), 50 Years of Archaeology in Southeast Asia. Essays in Honour of Ian Glover, Bangkok, River Books, 2010: 183-194. 
dans l'île dès le début du VII ${ }^{\mathrm{e}}$ siècle $^{31}$. Le nom même de «Śailendra», qui signifierait «roi de la montagne», a conduit Cœdès, en 1934, à l'interpréter comme une reprise des titres des anciens souverains du Funan ${ }^{32}$. Vingt ans plus tard, de Casparis déchiffre le toponyme «Varanara» dans une inscription de Plaosan à Java Central. Ce texte datable du IX ${ }^{\mathrm{e}}$ siècle mentionne également le nom d'un roi qui semblerait être le fondateur à Java de cette dynastie Śailendra. De Casparis rapproche ce toponyme de Naravaranagara, le Na-fu-na des sources chinoises, qui n'est autre que le nom de la capitale du Funan après l'abandon de Vyādhapura dans la seconde moitié du VI $\mathrm{V}^{\mathrm{e}}$ siècle. Il retrouve ce toponyme Naravara sur l'inscription voisine de Kelurak (782 EC), affirmant qu'il n'y a pas d'ambiguïté sur la référence à la capitale du Funan. Il lit par ailleurs dans l'inscription de Plaosan le titre adhirāja, inhabituel à l'époque à Java pour désigner les rois, alors que c'est justement le titre en usage pour les souverains du Funan installés à Vyādhapura ${ }^{33}$. Ajoutons que sur le plan chronologique, l'hypothèse se tient puisque qu'on sait maintenant qu'une importante rupture s'opère au Funan vers le milieu du VII $^{\mathrm{e}}$ siècle $^{34}$, période qui correspondrait à celle de l'émergence des Śailendras à Java. Ces hypothèses et conjectures sont toutefois loin de faire l'unanimité ${ }^{35}$.

En ce qui concerne le VIII ${ }^{\mathrm{e}}$ siècle, trois types de sources semblent faire état de velléités de conquête de la part du monde insulindien à l'encontre de la zone Jiaozhi (futur Viêt Nam) - Campā - Cambodge. Mentionnons tout d'abord un passage dans un texte soundanais tardif, la Carita Parahiyangan, dont des extraits ont été publiés initialement par Poerbatjaraka, qui raconte que le roi Sanjaya, dont on sait par ailleurs qu'il a régné à Java Central à partir de $732 \mathrm{EC}$, entreprend des conquêtes territoriales parmi lesquelles figure «kěmir», un toponyme qu'il est logique d'associer à l'actuel Cambodge ${ }^{36}$. Néanmoins, l'écart temporel entre le moment de ces conquêtes supposées et l'époque de rédaction du texte est tel que cette information doit

31. Boechari, "Preliminary Report on the Discovery of an Old-Malay Inscription at Sodjomerto", Majalah Ilmu-Ilmu Sastra Indonesia, oct., III, 2/3: 241-251 (réédité dans Boechari, Melacak Sejarah Kuno Indonesia Lewat Prasasti. Tracing Ancient Indonesian History through Inscriptions, Jakarta, KPG, FIB UI, EFEO, 2012: 349-360).

32. Cœdès, 1964: 168.

33. J.G. de Casparis, Prasasti Indonesia II. Selected Inscriptions from the $7^{\text {th }}$ to the $9^{\text {th }}$ Centuries, Bandung, Masa Baru, 1956: note 39 p. 184-5.

34. Manguin, 2009: 114.

35. Voir par exemple L.-C. Damais dans son compte rendu des deux ouvrages de De Casparis (1968: 421-422), ou encore Boechari (1966, 2012).

36. R. Ng. Poerbatjaraka, "De Batoe-Toelis bij Buitenzorg", TBG, LIX, 1919-21: 403 (380418). Plusieurs manuscrits de ce texte sont datés du XVII ${ }^{\text {e }}$ siècle (cf. Atja \& Edi S. Ekadjati, Carita Parahiyangan, Bandung, Yayasan Pembangunan Jawa Barat, 1989: 87-96). 
être traitée avec la plus grande précaution ${ }^{37}$. Selon les Annales vietnamiennes, en $767 \mathrm{EC}$ une partie de la région est envahie par des bandes venues du monde insulindien ${ }^{38}$, mais elles sont défaites par le gouverneur local. Sept ans plus tard au Campā, c'est une inscription sanskrite de Po Nagar à Nha Trang, alors escale pour les bateaux venant de l'archipel notamment ${ }^{39}$, qui raconte que «des hommes nés dans d'autres pays, des hommes vivant d'aliments plus horribles encore que les cadavres, effrayants, entièrement noirs et maigres, terribles et méchants comme la mort, venus sur des navires» désacralisent et brûlent le temple. Une autre inscription, datée 787 EC, plus au sud dans la plaine de Phan Rang cette fois, mentionne que «les armées de Java, venues sur des vaisseaux» brûlent un autre temple ${ }^{40}$. À partir de ces deux textes et d'autres sources épigraphiques, Arlo Griffiths apporte dans ce dossier un nouvel éclairage sur les rapports entre le «Java» des inscriptions et la péninsule indochinoise au tournant du IX ${ }^{\mathrm{e}}$ siècle. En contrepoint à ces conflits, les historiens de l'art décèlent dans l'architecture cam le développement d'un moment «indo-javanais» qui ne peut être antérieur au $\mathrm{VIII}^{\mathrm{e}}$ siècle, et se situe probablement entre la seconde moitié du $\mathrm{IX}^{\mathrm{e}}$ siècle et le début du $\mathrm{X}^{\mathrm{e}}$ siècle, moment qui traduit des contacts culturels étroits ${ }^{41}$.

En 802 EC au Cambodge, Jayavarman II organise une cérémonie visant à se libérer de la domination de «Java» et une inscription du $\mathrm{XI}^{\mathrm{e}}$ siècle nous apprend d'ailleurs qu'il est venu de «Java» pour régner à Indrapura ${ }^{42}$. Cœdès rapproche cette situation de dépendance du Cambodge vis-à-vis de «Java» d'une source arabe du début du $\mathrm{X}^{\mathrm{e}}$ siècle, le Voyage du marchand arabe Sulaymân en Inde et en Chine, qui relate un conflit entre le roi khmer et le roi de Jâwaga :

«[le roi khmèr s'exprime] 'Je désire voir devant moi, sur un plat, la tête du Mahârajâ, roi de Jâwaga' (...) le propos parvint à la connaissance du Mahârajâ (...)[ce dernier prescrivit

37. Sur ce point, voir L.-C. Damais, "Bibliographie. Compte rendu de l'ouvrage de Poerbatjaraka, Riwayat Indonesia, 1952”, BEFEO, 48(2), 1957 : 635sq.

38. Voir l'article de C. Salmon dans ce volume sur le terme employé à cette époque pour désigner le monde insulindien.

39. W.A. Southworth, $2004: 226$ (209-233).

40. Cœdès, 1964 : 173.

41. Philippe Stern, L'art du Champa (ancien Annam) et son évolution, Toulouse, Les Frères Douladoure, 1942 : 68; B.P. Groslier, Indochine. Carrefour des Arts, Paris, Editions Albin Michel, 1961 : 133-6, 141, 143; J. Guy, "Artistic Exchange, Regional Dialogue and the Cham Territories", in A. Hardy, M. Cucarzi \& P. Zolese (eds.), Champa and the Archaeology of Mỹ Son (Vietnam), Singapore, NUS Press, 2009: 147-151 (107-126). À l'inverse, le Candi Pari à Java-Est (XIV siècle) montrerait une influence cam dans son architecture (N.J. Krom, Inleiding tot de Hindoe-Javaansche Kunst, 's-Gravenhage, M. Nijhoff, 1923, vol. II: 185).

42. B.P. Groslier, 1961 : 88-9; Cœdès, $1964: 183-4$. Cette question est reprise par A. Griffiths dans le présent dossier. 
de] faire préparer mille navires de moyenne grandeur, de les équiper, de mettre à bord de chacun d'eux des armes et des troupes vaillantes (...) il déclara ouvertement qu'il désirait faire un voyage d'agrément dans les îles de son royaume (...) celui-ci s'embarqua et avec sa flotte et ses troupes fit route à destination du royaume de Khmèr (...). [une fois dans la capitale] Il fit alors couper la tête au roi du Khmèr. Puis il s'approcha du ministre khmèr et lui dit '(...). Cherche maintenant quelqu'un qui puisse faire un bon roi après ce fou, et mets-le à la place de celui-ci'. Le Mahârâja partit sur l'heure pour retourner dans son pays (...) et il fit mettre devant lui le plat contenant la tête du roi du Khmèr. (...) Le Mahâraja fit ensuite laver et embaumer la tête du roi du Khmèr; on la mit dans un vase et on l'envoya au roi qui avait remplacé sur le trône du Khmèr le souverain décapité (...). Depuis ce moment, les rois du Khmèr, tous les matins, en se levant, tournent le visage dans la direction du pays de Jâwaga, s'inclinent jusqu'à terre et s'humilient devant le Mahâraja pour lui rendre hommage.» 43

Cœdès ajoute toutefois que ce passage pourrait être le souvenir romancé d'une attaque de Śrīvijaya à la fin du VII siècle $^{44}$. Ajoutons en ce qui concerne le $\mathrm{IX}^{\mathrm{e}}$ siècle qu'une influence javanaise est perceptible dans l'art d'Angkor, notamment dans le style des kala et makara, ainsi que sur certains haut-reliefs 45 .

À Java, l'inscription en vieux-javanais de Kuti (Java Est, 840 EC) donne une liste des pays d'origine de wargge dalěm (domestiques du palais). Parmi eux figurent des gens du Campā (cémpa) et du Cambodge $(k m i r){ }^{46}$. À la même époque au Campā, c'est probablement toujours le port de Nha Trang qui domine dans les relations avec 1'Archipel, alors qu'entre la fin du VIII ${ }^{\mathrm{e}}$ siècle et le début du $\mathrm{IX}^{\mathrm{e}}$ siècle il était en concurrence pour ces échanges avec le port de Long Bien dans la vallée de la Rivière Rouge ${ }^{47}$. Sur ces contacts anciens, une inscription du Campā du début du $\mathrm{X}^{\mathrm{e}}$ siècle rapporte que par deux fois, sous deux souverains différents (qui ont régné entre la fin du IX ${ }^{\mathrm{e}}$ et le début du $\mathrm{X}^{\mathrm{e}}$ siècle), un certain Po kluñ Pilih Rājadvāra, dignitaire apparenté à la maison royale, s'est rendu en ambassade à Java ${ }^{48}$.

43. G. Ferrand (trad. \& éd.), Voyage du marchand arabe Sulaymân en Inde et en Chine rédigé en 851, suivi de remarques par Abû Zayd Hasan (vers 916), Paris, Editions Bossard, 1922: 99-102.

44. Coedès, $1964: 177$.

45. Styles de Kulên et de Preah Ko, Bakong (Groslier, 1961 : 91, 97-99).

46. H.B. Sarkar, Corpus of the Inscriptions of Java, Calcutta, Firma K.L. Mukhopadhyay, 1971, vol. 1: 76-99. Ce document est toutefois à traiter avec précaution dans la mesure où il s'agit d'une copie réalisée à l'époque de Majapahit. Dans son répertoire onomastique (Paris, PEFEO LXVI, 1970), dont le corpus comprend les inscriptions javanaises antérieures à 928 EC, Damais ne relève le terme campa qu'à cinq reprises (dans des inscriptions datées entre 762 Ś et 831 Ś, soit 840-909 EC) et kmir à six reprises (dans des inscriptions datées entre 762 et 844 S, soit 840-922 EC).

47. W.A. Southworth, 2004: 227-8.

48. E. Huber, «Études indochinoises», BEFEO, 11, 1911: 301, 303, 309 (259-311). L'interprétation que fait Huber d'un pèlerinage réalisé à Java pour y apprendre la sorcellerie (ou la science magique) est aujourd'hui contestée (cf. la contribution de A. Griffiths dans ce dossier). 
On retrouve l'ethnonyme kmir dans une inscription de Java Central de la première moitié du $\mathrm{X}^{\mathrm{e}}$ siècle (Wuruḍu Kidul, $922 \mathrm{EC}$ ), qui reprend un jugement suite à un litige dans lequel un individu saisit la justice parce qu'il a été traité de «fils de Khmer» (vka kmir), ce qu'il considère comme une insulte ${ }^{49}$.

Les annales de la dynastie Song (960-1279) rapportent qu'au royaume de «San-bo-tsai» (Śrīvijaya), des « esclaves de Pulo Condore» y sont musiciens et chanteurs, et qu'en 988 un ambassadeur apporte un tribut à la cour de Chine avant de se rendre au Campā avec son navire ${ }^{50}$. Cette mention permet de mettre l'accent ici sur le rôle des communautés étrangères dès cette époque dans les relations entre le monde insulindien et la péninsule indochinoise. Il est en effet probable qu'une bonne partie de ces envoyés au service d'entités politiques du monde insulindien étaient d'origines chinoise, sud-asiatique ou moyenne-orientale, et que certains d'entre eux étaient métis ${ }^{51}$. Des marchands étrangers et métis ont aussi très probablement joué le rôle d'intermédiaires dès cette époque, à travers des réseaux reliant diverses régions d'Asie du Sud-Est et leur pays d'origine ${ }^{52}$. Ce sont justement surtout les sources chinoises et moyennes-orientales qui permettent encore de reconstituer les anciennes routes maritimes entre la péninsule indochinoise et le monde insulindien ${ }^{53}$. Geoff Wade reprend cette question sur la longue durée dans le présent dossier.

$\mathrm{Au} \mathrm{X}^{\mathrm{e}}$ siècle, le Campā comprend plusieurs ports accueillant probablement des marchands de l'Archipel, entre le port d'Amarendrapura au nord et Phan Rang au sud ${ }^{54}$. Il faut noter en particulier 1'archipel de $\mathrm{Cu}$ Lao Cham, déjà cité plus haut, lieu de transbordement qui a livré de gros fragments de poteries glaçurées du Moyen-Orient datables des $\mathrm{IX}^{\mathrm{e}}-\mathrm{X}^{\mathrm{e}}$ siècles, poteries qui reflètent vraisemblablement des contacts avec l'Archipel

49. H.B. Sarkar, Corpus of the Inscriptions of Java, up to 928 A.D., Calcutta, Firma K.L. Mukhopadhyay, 1971-2, vol. 2: 198-206; Boechari, 2012: 242.

50. W.P. Groeneveldt, Historical Notes on Indonesia and Malaya compiled from Chinese Sources, Djakarta, C.V. Bhratara, 1960 (1 ère éd., 1877): 63, 65.

51. Voir par exemple le cas des intermédiaires entre Śrīvijaya et la Chine étudié par C. Salmon ("Srivijaya, la Chine et les marchands chinois ( $\mathrm{X}^{\mathrm{e}}-\mathrm{XII}^{\mathrm{e}} \mathrm{s}$.). Quelques réflexions sur l'empire sumatranais", Archipel, 63, 2002 : 57-78).

52. Pour deux synthèses récentes sur cette question, voir C. Salmon, «Les Persans à l'extrémité orientale de la route maritime (II ${ }^{\mathrm{e}}$ A.E.-XVII ${ }^{\mathrm{e}}$ siècle)», Archipel, 68, 2004 : 2358; Geoff Wade, "An Early Age of Commerce in Southeast Asia, 900-1300 CE", JSEAS, 40(2), 2009: 221-265.

53. On peut ajouter un exemple de source d'origine différente, tel ce routier arménien de la mer de Chine du début du XII ${ }^{\mathrm{e}}$ siècle. Une communauté arménienne est installée à Zaytun (= Quanzhou) au moins dès le tout début du XIVe siècle, puisqu'on sait qu'elle y finance la construction d'une église pour le compte de Rome (K. Kévonian, «Un itinéraire arménien de la mer de Chine», in C. Guillot (éd.), Histoire de Barus, Sumatra. Le site de Lobu Tua. I : Études et Documents, Paris, Cahier d'Archipel 30, 1998 : 35-118).

54. K.R. Hall, 2011: 77. 
à l'époque. Il convient d'y ajouter le port de Cua Dai Chiem sur la côte en face de $\mathrm{Cu}$ Lao Cham ${ }^{55}$.

À Java même, deux inscriptions en vieux-javanais du delta du fleuve Brantas, dans l'est de l'île (Patakan et Cane), datées 1021 EC, mentionnent la présence de gens du Campā et des «Kmir» ${ }^{56}$.

Dès la fin du $\mathrm{X}^{\mathrm{e}}$ siècle, après la libération définitive du Dai Viet de la domination chinoise en 939 EC, les chroniques officielles vietnamiennes font état d'une ouverture au grand commerce, avec une fréquentation de la capitale Hoa Lu et de son port de Bo Hai par des marchands venant d'autres régions d'Asie du Sud-Est, en particulier de Java ${ }^{57}$. Les mêmes sources décrivent une situation similaire pour le port de Van Don, dans le delta de la Rivière Rouge, à partir du milieu du XII' siècle. Van Don au nord, et, au sud, le nouveau port de Thi Nai (Quy Nhon dans la province actuelle de Binh Dinh) à Vijaya au Campā, semblent apparaître en même temps, à une époque d'accroissement du grand commerce entre Java Est et la Chine du Sud ${ }^{58}$. Un peu plus tard, dans les années 1170, Fan Chengda, un lettré fonctionnaire de Chine rapporte que la côte du Dai Viet est une zone de rencontre entre les «infâmes trafiquants du sud de l'empire» et «les marchands étrangers des pays barbares $\gg 59$.

Une source chinoise compilée au XII ${ }^{\mathrm{e}}-\mathrm{XIII}{ }^{\mathrm{e}}$ siècle mentionne que le Campā est en relation, sans doute commerciale, avec «San-fo-qi » (probablement Śrīvijaya) et Butuan aux Philippines ${ }^{60}$.

Au Cambodge, une inscription de Jayavarman VII datée 1191 EC rapporte que, parmi d'autres souverains, le roi de Java «porte chaque jour avec piété l'eau des ablutions », le tribut d'eau étant très probablement à l'époque un signe d'allégeance ${ }^{61}$. Ce passage, certainement destiné à magnifier le souverain d'Angkor, montre que Java était bien présente dans la vision politique du monarque.

55. W.A. Southworth, 2004: 228; Andrew Hardy, "Eaglewood and the Economic History of Champa and Central Vietnam", in A. Hardy, M. Cucarzi \& P. Zolese (eds.), Champa and the Archaeology of Mỹ Son (Vietnam), Singapore, NUS Press, 2009: 108 (107-126).

56. J.L.A. Brandes, Oud-Javaansche Oorkonden Nagelaten Transscripties, Batavia, Albrecht \& Co., 's-Gravenhage, M. Nijhoff, VBG deel LX, 1913: 120-8, no. LVIII et LIX; N.J. Krom, Hindoe-Javaansche Geschiedenis, 's-Gravenhage, M. Nijhoff, 1931: 264.

57. K.W. Taylor, "The Early Kingdoms", in N. Tarling (ed.), The Cambridge History of Southeast Asia. I: From Early Times to c. 1500, Cambridge, Cambridge Univ. Press, 1999 (1ère éd., 1992): 145 (137-182).

58. M. Shiro, "Dai Viet and the South China Sea Trade: From the $10^{\text {th }}$ to the $15^{\text {th }}$ Century", Crossroads, 12(1), 1998: 14 (1-34).

59. J.K. Whitmore, "The Rise of the Coast: Trade, State and Culture in Early Đại Việt", JSEAS, 37(1), 2006: 110, 111 (103-122).

60. G. Wade, 2009: 242, 258 (221-265).

61. G. Codès, “La stèle du Prah Khằn d'Añkor”, BEFEO, 41, 1941: 267-8, 299 (255-302). 
Au début du XIII ${ }^{\mathrm{e}}$ siècle, c'est toujours le port de Van Don qui représente la plaque tournante principale du commerce entre le Dai Viet et 1'Archipel, et il le restera jusqu'au XIV ${ }^{\mathrm{e}}$ siècle. Au XIII ${ }^{\mathrm{e}}$ siècle, Thi Nai à Vijaya au Campā, reste le port cam principal servant d'escale entre l'Archipel et la Chine et deviendra même le port intermédiaire majeur de toute la péninsule pour ce commerce jusqu'aux premières décennies du $\mathrm{XV}^{\mathrm{e}}$ siècle ${ }^{62}$.

Maspero mentionne une inscription du Campā datée 1306 EC rapportant qu'une «princesse, fille du roi de Java, est venue du Yavadvipa et se nomme la reine Tapasi $"{ }^{63}$. En 1318 , le roi cam Che Nang, vaincu par les Vietnamiens, se réfugie à Java, une réaction logique s'il s'agit d'un fils de la reine Tapasi mentionnée ci-dessus ${ }^{64}$. À la même époque, le Dai Viet continue d'entretenir des relations avec 1'Archipel. On apprend ainsi que le frère d'un général du Dai Viet s'exprimait dans leur langue avec les envoyés de Temasek (actuel Singapour), langue qui pouvait être du malais, du persan ou de l'arabe 65 .

Le Deśawarnana, composé en 1365, inclut le Campā, le Cambodge et le Yawana (Dai Viet) parmi les pays «protégés» par le souverain de Majapahit à Java Est, ou «amis» de ce royaume. Ces trois pays apparaissent également ensemble dans un autre passage du texte, où la gloire du souverain, ainsi que la présence de religieux illustres, attirent des visiteurs de la Chine à l'Inde, visiteurs qui font le voyage avec les marchands ${ }^{66}$. L'influence de la péninsule indochinoise à Majapahit dès le $\mathrm{XIV}^{\mathrm{e}}$ siècle est encore perceptible à travers les céramiques originaires de cette région retrouvées dans les fouilles de Trowulan, l'ancienne capitale du royaume ${ }^{67}$. Trois siècles plus tard, Dampier note qu'un marchand anglais a acheté 100000 bols de basse qualité à Thang Long (Hanoi), cargaison revendue pratiquement en totalité sur la côte ouest de Sumatra avec un énorme profit ${ }^{68}$. Cet aspect des relations, à savoir la diffusion des céramiques vietnamiennes dans le monde

62. K.R. Hall, 2011: 241, 242.

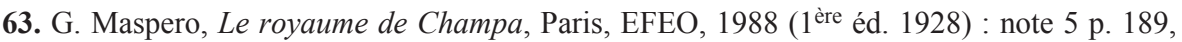
199.

64. Maspero, 1988: 197-9.

65. Li Tana, "A View from the Sea: Perspectives on the Northern and Central Vietnamese Coast”, JSEAS, 2006: 91 (83-102).

66. Th.G.Th. Pigeaud, Java in the $14^{\text {th }}$ century: A study in cultural history/The NāgaraKërtāgama by Rakawi Prapañca of Majapahit, 1365 A. D. The Hague, M. Nijhoff (KITLV, Translation Series 4), 1960, I: 12, 64; III: 18, 98; 1962, IV: 35, 272-3 (5 vol.); S. Robson (transl.), Deśawarnana (Nãgarakrtāgama) by Mpu Prapañca, Leiden, KITLV Press, 1995: 34,85 .

67. Dupoizat, 2003.

68. William Dampier (1699) cité par A. Reid, Southeast Asia in the Age of Commerce, 14501680, vol. I, New Haven/London, Yale Univ. Press, 1988: 105. 
insulindien pendant plus de trois siècles, est examiné dans ce dossier par Marie-France Dupoizat.

Majapahit et la péninsule indochinoise, c'est aussi l'histoire très populaire à Java de la fameuse Putri Cempa, rapportée dans plusieurs textes littéraires javanais, personnage lié à l'introduction de l'islam à Java. S'il n'est pas possible de reprendre ici cette question en détail, à notre connaissance, il n'y a pour l'instant aucune source cam qui confirme cette légende. Pour s'en tenir à un seul texte, le Babad Tanah Jawi, la chronique officielle du royaume de Mataram, dont nous n'avons examiné qu'un résumé d'un manuscrit publié ${ }^{69}$, cette histoire comporte trois épisodes à l'époque du souverain Brawijaya de Majapahit ${ }^{70}$. Rêvant qu'il épouse une princesse du Campā (Cempa dans le texte), Brawijaya envoie une délégation au Campā pour transmettre son souhait. Elle revient avec la fille aînée du roi du Campā, Darawati, que Brawijaya épouse ${ }^{71}$. Au Campā, un musulman nommé Makdum Ibrahim Asmara (beau-frère du nouveau roi du Campā et de Darawati) a deux fils, Raden Rahmat et Raden Santri (qui sont donc musulmans). Raden Rahmat souhaite retrouver sa tante à Java et y part avec son frère ainsi qu'avec Raden Burereh, le fils du roi. Tous trois passent un an au palais de Majapahit et prennent chacun une épouse. Raden Rahmat s'installe à Surabaya, dans le quartier d'Ampel Denta, où il devient imam de la mosquée à la demande de Brawijaya ${ }^{72}$. Deux de ses nombreux disciples

69. Nous avons consulté la traduction en indonésien parue en 2004 (S.D. Damono \& S. Sondakh, eds. trad., Babad Tanah Jawi, Jakarta, Amanah-Lontar, 2 vol.), d'un résumé de l'édition en javanais en 31 volumes parue en 1939 (éditée par R. Ng. Yasadipura et publiée par Balai Pustaka). Il y a probablement autant de versions différentes concernant Putri Cempa qu'il y a de manuscrits, qui sont conservés dans au moins cinq collections (S.D. Damono \& S. Sondakh, eds. trad., $2004: \mathrm{xv}$ ). Pour une brève revue déjà ancienne, mais encore utile, des études sur ce texte, voir H.J. de Graaf, "Later Javanese Sources and Historiography", in Soedjatmoko et al. (eds.), An Introduction to Indonesian Historiography, Ithaca, New York, Cornell Univ. Press, 1965 : 119-122 (119-136).

70. Les avis divergent sur l'identité et l'époque du règne de ce souverain. J. Noorduyn le place au début du XVI ${ }^{\mathrm{e}}$ siècle ("Majapahit in the Fifteenth Century", BKI, 134(2/3), 1978: 243 (207-274), alors que H. Djafar l'identifie avec Bhre Krtabhūmi et situe son règne entre 1468 et 1478 (cf. Masa Akhir Majapahit. Girîndrawarddhana dan Masalahnya, Jakarta, Komunitas Bambu, 2009).

71. Le texte malais Hikayat Hasanuddin comporte également un épisode relatant le mariage entre un roi de Majapahit et une princesse du Campā (Malay Concordance Project http://mcp.anu.edu.au/-. Jan Edel, Hikajat Hasanoeddin, Meppel, Ten Brink, Akademisch Proefschrift Rijksuniversiteit te Utrecht,1938: 64-27, 64-30, 68-28). Selon la Babad Meinsma, elle apporte un gong, un carosse et une charrette qui feront plus tard partie du trésor royal de Mataram (H.J. de Graaf \& Th. G. Th. Pigeaud, De eerste Moslimse vorstendommen op Java: studiën over de staatkundige geschiedenis van de 15de en 16de eeuw, 's-Gravenhage, M. Nijhoff, VKI 69, 1974: 20).

72. Selon une autre source, Raden Rahmat aurait été accueilli à Ampel Denta par un Chinois "chef des marchés» (Pecat tanda) au service de Majapahit (D. Lombard \& C. Salmon, «Islam et Sinité», Archipel, 30, 1985 : 74 (73-94)). Parmi les premiers chercheurs à 
sont fort connus dans l'histoire de l'islamisation de l'île, il s'agit de Sunan Bonang et de Sunan Giri ${ }^{73}$. Quant à Raden Santri et Raden Burereh, ils s'installent à Gresik. Le troisième épisode intervient lorsque Brawijaya tombe gravement malade. Pour guérir, la reine Darawati lui conseille de coucher avec Putri Wandan Kuning, l'une de ses servantes également originaire du Campā. Celle-ci lui donne un fils que Brawijaya confie à l'un des fonctionnaires du palais en lui ordonnant de le tuer lorsqu'il aura atteint l'âge de huit ans. Sur le site de Trowulan, l'ancienne capitale de Majapahit, on peut encore voir une tombe que la tradition locale attribue à Putri Cempa. Le millésime figurant dans l'inscription de la stèle correspond à 1448/9 EC ${ }^{74}$. Raden Rahmat, qui deviendra Sunan Ampel, est traditionnellement considéré comme le plus important des neuf saints (Wali Sanga) ayant islamisé l'est et le centre de Java à partir de la seconde moitié du XVe siècle ${ }^{75}$.

Une autre tradition liée à l'époque de Majapahit, moins connue cette fois, est celle d'une attaque de la côte orientale de Java par des troupes siamoises et khmères alliées à Sukadana (Bornéo), attaque qui aurait été repoussée par un personnage originaire du massif du Tengger. En récompense, il aurait reçu le territoire de Blambangan ${ }^{76}$.

Dans le texte malais Sulalat al-Salatin, les Cam apparaissent dans le récit du royaume de Melaka juste après la conversion de son souverain à l'islam. La réorganisation du protocole palatin donne une place de choix à un certain nakhoda $\mathrm{Cempa}^{77}$. On retrouve les Cam à propos des relations entre le monde insulindien et la péninsule indochinoise à l'époque de Majapahit, avec

s'être intéressés à la question, citons P.J. Veth qui consacre plusieurs pages à "Ratoe Poetri Tjampa" et à Raden Rahmat dans son Java, geographisch, ethnologisch, historisch, Haarlem, De Erven F. Bohn, 1896, vol. I: 233sq.. Pour un examen de sources littéraires de Java associant la diffusion de l'islam dans l'île et le Campā, voir Manguin, 1979 : 261-4.

73. À propos de Sunan Giri, on dispose de quelques informations sur sa mère nourricière, Nyai Pinatih, une Chinoise originaire de Palembang (Lombard \& Salmon, 1985 : 74). Au tournant du XVI $\mathrm{X}^{\mathrm{e}}$ siècle, cette musulmane peut-être d'origine khmère, est active à Java-Est pour la promotion de l'islam. Elle est probablement un temps syahbandar de Gresik, propriétaire d'une flotte de commerce, envoyant des navires jusqu'au Cambodge (T.S. Raffles, II, 1994 : 115; M.A.P. Meilink-Roelofsz, Asian Trade and European Influence in the Indonesian Archipelago between 1500 and about 1630, The Hague, M. Nijhoff, 1962 : 108). Selon De Graaf \& Pigeaud (1974 : note 178 p. 285), son époux était probablement khmer.

74. C'est Brandes qui en publie le premier une lecture correcte, après une lecture fautive publiée par Raffles (NBGKW, XXIV, $1886: 42)$.

75. Th.G.Th. Pigeaud \& H.J. de Graaf, Islamic states in Java, 1500-1700, The Hague, M. Nijhoff, VKI 70, 1976: 5, 16. Voir aussi T.S. Raffles, The History of Java, Kuala Lumpur, Oxford Univ. Press, vol. II, 1994 (1 ère éd., 1817) : 124. Ses deux disciples, Sunan Bonang et Sunan Giri, font également partie des Wali Songo.

76. De Graaf \& Pigeaud, 1974 : 192, note 174 p. 284.

77. A. Samad Ahmad (ed.), Sulalatus Salatin, Kuala Lumpur, Dewan Bahasa dan Pustaka, 1986 (1 ère éd., 1984): 75. 
la visite d'un certain Pau Gama, roi de Bal, à la cour du royaume javanais, où le souverain local lui donne comme épouse l'une de ses filles, Raden Galuh Ajeng. Pau Gama retourne au Campā au moment où la princesse est enceinte. Raja Jakanak, le fils né de cette union, part au Campā, où son père le fait roi. La chronique continue en racontant que c'est au moment où Pau Kubah, le successeur de Raja Jakanak, refuse de donner sa fille en mariage au roi de $\mathrm{Kuci}^{78}$ (Dai Viet) que ce dernier attaque et conquiert la capitale ${ }^{79}$.

Ce conflit est bien connu par ailleurs, puisqu'il s'agit de la prise de Vijaya, la capitale du royaume du Campā, en $1471^{80}$. L'événement provoque le déplacement de la capitale au sud, dans la région de Phan Rang, tandis que Van Don au Dai Viet devient le port d'escale majeur de la péninsule indochinoise entre 1'Archipel et la Chine ${ }^{81}$. Des Cam s'enfuient en direction du monde insulindien. Le Sulalat al-Salatin identifie deux princes cam, respectivement Syah Indera Berma, qui se réfugie à Melaka, et Syah Pau Ling, qui se réfugie à Aceh où, le texte nous précise, il est à l'origine des souverains d'Aceh ${ }^{82}$. Un autre élément sans doute beaucoup plus ancien relie Aceh et le Campā, ce sont les deux langues, dont les similitudes ont donné lieu à un certain nombre d'études linguistiques ${ }^{83}$. La présence cam à Aceh a peut-être imprimé sa marque dans le paysage puisque trois toponymes de la pointe nord de Sumatra, encore en usage aujourd'hui, comportent le terme «Cempa» ${ }^{84}$. Il est toutefois plus probable que ce mot

78. Kuci tire son origine du terme ancien Jiaozhi en chinois, rendu Giao Chi en vietnamien, qui sera ensuite emprunté par les Portugais pour donner Cochin-China, que l'on retrouve déjà chez Tomé Pires.

79. A. Samad Ahmad (ed.), 1986: 163-4.

80. Dans ses Légendes historiques des Chames, Aymonier raconte : «En l'année du serpent les Annamites amenèrent des troupes entourer la capitale Bal Thuh [...] A Bal Thuh, leurs têtes furent amoncelées en pyramides hautes comme des montagnes.» (cité par Georges Maspero, «Le royaume de Champa. Chapitre IX», T'oung Pao, Second Series, Vol. 12, No. 5 (1911), pp. 589-626, à la note 5 page 603).

81. K.R. Hall, 2011: 250.

82. A. Samad Ahmad (ed.), 1986: 163-4. La conquête de la capitale du Campā et la fuite de Cam vers l'Archipel sont mentionnées dans un autre texte malais, le Hikayat Hasanuddin (Malay Concordance Project, Jan Edel, Hikajat Hasanoeddin, Meppel, Ten Brink, Akademisch Proefschrift Rijksuniversiteit te Utrecht, 1938: 74-4). Une tradition sundanaise tardive, le Sejarah Banten rante-rante, affirme qu'après le départ de Raden Rahmat et de ses compagnons, le «Cěmpa» est dévasté par le roi de Koci (Manguin, 1979 : note 1 p. 262).

83. Au-delà de la parenté linguistique, Thurgood (1999:20-21, 42-43) avance que les Acihais sont les descendants de Cam qui auraient quitté la partie centrale de la côte du Vietnam au X $\mathrm{X}^{\mathrm{e}}-\mathrm{XI}^{\mathrm{e}}$ siècle (après le sac d'Indrapura, $982 \mathrm{EC}$ ) pour s'installer à Aceh. Les auteurs remercient Jérôme Samuel pour cette information. Nous pensons néanmoins qu'il convient de traiter avec la plus grande prudence ce genre d'hypothèse aussi précisemment datée, tant qu'elle n'est pas confirmée par d'autres sources.

84. Il s'agit de deux villages sur le territoire actuel d'Aceh, que sont Pantai Cempa, Kecamatan Tamiang Hulu, Kabupaten Aceh Tamiang, et Cempa, Kecamatan Blangkejeren, 
soit d'origine botanique, comme de nombreux toponymes à Sumatra. En effet, à Sumatra, "campa » désigne Michelia champaca (famille des Magnoliacées), un arbre connu pour ses fleurs jaunes ou blanches odoriférantes ${ }^{85}$.

Le Sulalat al-Salatin poursuit en racontant qu'à Melaka, Syah Indera Berma entre en faveur auprès de Sultan Mansur Syah, et qu'à la demande du souverain, lui-même, son épouse et sa suite se convertissent à l'islam, avant qu'il ne soit fait ministre. Selon le texte malais, ils sont les ancêtres de tous les Cam de Melaka ${ }^{86}$. On retrouve, dans certaines versions de la chronique, mention d'un Cam capitaine de navire, qui participe à l'enlèvement de la fille du bendahara de Pahang qui deviendra l'épouse de Sultan Mahmud ${ }^{87}$.

Les rapports commerciaux entre le monde insulindien et la péninsule indochinoise se précisent avec la multiplication des sources portugaises. Au début du $\mathrm{XVI}^{\mathrm{e}}$ siècle, le Campā ne dispose pas de port pouvant recevoir de grandes jonques, mais de nombreux lanchara naviguent jusqu'à Pahang. Le Campā est alors surtout réputé pour son bois d'aloès, aussi nommé bois d'aigle ou calambac (Aquilaria sp. ${ }^{88}$, le meilleur de la région, ainsi que pour son or, à propos duquel Pires précise qu'il s'agit également du meilleur de la région. Ces deux produits sont négociés jusqu'à Melaka, de même que le poisson salé séché et le riz. Dans l'autre sens, on y trouve à acheter de l'or provenant du pays minangkabau ${ }^{89}$. De son côté à l'époque, le Dai Viet envoie rarement ses navires à Melaka, mais ses négociants s'y rendent sur des jonques chinoises. Melaka lui fournit surtout de grandes quantités de soufre provenant de l'île de Solor ${ }^{90}$. À Melaka, Vietnamiens et Cam sont

Kabupaten Gayo Lues. Le troisième est le village de Cempa, qui se situe aujourd'hui dans la province de Sumatra Nord, Kecamatan Hinai, Kabupaten Langkat.

85. On peut citer d'autres toponymes «Cempa» dans le monde insulindien : Pulau Cempa, Kabupaten Lingga, Province de Kepulauan Riau; Kampung Cempa, Desa Cilangkap, Kec. Kalanganyar, Kab. Lebak, Province de Banten; Desa Cempa, Kecamatan Cempa, Kabupaten Pinrang, Province de Sulawesi Selatan; Lubok Chepa, sur la rivière Nerus, près de Kampung Lubok Buaya dans l'État de Terengganu en péninsule malaise, ou encore, toujours en péninsule, Pengkalan Chepa, petite localité de Kelantan près Kota Bharu.

86. A. Samad Ahmad (ed.), 1986: 165.

87. W.G. Shellabear (éd.), Sejarah Melayu, Kuala Lumpur, Penerbit Fajar Bakti, 1994 (1 ère éd. en jawi, 1896): 168-171; John Leyden (éd), John Leyden's Malay Annals, Kuala Lumpur, MBRAS Reprint 20, 2001 (1ère éd., London, 1821): 293-302; Muhammad Haji Salleh (ed.), Sulalat al-Salatin, ya'ni Perteturun Segala Raja-Raja (Sejarah Melayu), Kuala Lumpur, Dewan Bahasa dan Pustaka, Yayasan Karyawan, 2009 (1 1 ère éd., 1997): 182-186.

88. Pour un point récent sur le commerce du bois d'aigle au Campā, voir Hardy, 2009 : 116-118. 89. A. Cortesao (ed. \& trad.), The Suma Oriental of Tomé Pires, London, Hakluyt Society, 1944, I: 112-3, II: 275. Ce commerce de l'or pourrait expliquer, au moins partiellement, la présence de gens de Minangkabau dans la péninsule indochinoise, présence furtive dans plusieurs textes malais. Les chercheurs d'or «malais» actifs au Cambodge au début du XVII ${ }^{\mathrm{e}}$ siècle pourraient bien être des Minangkabau (voir infra en ce qui concerne la Nam Noy).

90. Pires, 1944, I : 114-5; II : 203. 
sous l'autorité d'un même syahbandar, qui est également responsable des Chinois et des gens de Ryukyu ${ }^{91}$. Ajoutons que des marchands du Cambodge fréquentent aussi la ville ${ }^{92}$.

À la suite de la prise de Melaka par les Portugais en 1511, une partie des marchands musulmans de la ville s'enfuient pour s'installer dans des ports de la région, ainsi qu'au Cambodge, où ils sont perçus et s'identifient peutêtre comme Malais ${ }^{93}$. Au Cambodge, ils ne sont probablement pas les premiers «Malais » à s'installer ${ }^{94}$ et s'attirent dès le XVI ${ }^{\mathrm{e}}$ siècle la confiance des dirigeants, puisque des Malais font partie de la garde personnelle du roi Paramarājā III (1566-1576) ${ }^{95}$. C'est cette communauté malaise du Cambodge, vue à travers le prisme des fonctionnaires coloniaux français au $\mathrm{XIX}^{\mathrm{e}}$ siècle, qu'aborde la contribution de Nicolas Weber dans ce dossier. La prise de Melaka ne semble pas interrompre le commerce entre le Cambodge et la péninsule malaise, puisqu'une version des chroniques royales du Cambodge indique l'envoi de jonques vers Mariyūv (Malayū) pour y acheter des canons et des fusils 96 . On sait par ailleurs que des marchands cochinchinois et cam fournissent de grandes quantités de soie à Melaka à la fin du siècle ${ }^{97}$.

Dès le $\mathrm{XVI}^{\mathrm{e}}$ siècle en ce qui concerne les Portugais et les Espagnols, et dès le siècle suivant pour d'autres Européens, il faut intégrer la composante occidentale dans le paysage des relations entre le monde insulindien et la péninsule indochinoise. Il n'est bien sûr pas possible d'aborder ce sujet ici et nous ne pouvons que renvoyer aux nombreuses études publiées.

Le rôle des Japonais est également à considérer dès cette époque au moins, et jusqu'en 1635, après quoi il leur est interdit de sortir de leur pays. On sait par exemple qu'à la fin du XVI ${ }^{\mathrm{e}}$ siècle des mercenaires japonais se trouvent à bord de navires espagnols partant de Manille pour attaquer le Cambodge, alors que d'autres sont au service de la cour du Cambodge elle-

91. Pires, 1944, I : 126 II : 265.

92. Pires, 1944, II : 268.

93. A. Reid, Southeast Asia in the Age of Commerce, 1450-1680, vol. II, New Haven/London, Yale University Press, 1993: 126-128; A. Reid, "Economic and Social Change, c. 14001800 ", in N. Tarling (ed.), The Cambridge History of Southeast Asia, Cambridge, Cambridge University Press, vol. I (part two), 1999 (1 ère éd., 1992): 137 (116-163).

94. Cependant, ni Mak Phoeun (1995b : 1271, 1285), ni Mohamad Zain bin Musa (2001 : 4) ne font remonter l'histoire de cette communauté avant le XVI ${ }^{\mathrm{e}}$ siècle.

95. Mak Phoeun, Histoire du Cambodge de la fin du XVIe siècle au début du XVIIIe siècle, Paris, Presses de l'Ecole française d'Extrême-Orient, $1995: 321$.

96. Khin Sok, Chroniques Royales du Cambodge (de Bañā Yãt à la prise de Lañvaek) (de 1417 à 1595), Paris, École française d'Extrême-Orient, Coll. de textes et documents sur l'Indochine XIII, $1988: 285$.

97. Shiro, $1998: 21$. 
même ${ }^{98}$. À cette époque, des gens originaires du monde insulindien, ainsi que des Cam musulmans font partie des plus importantes minorités marchandes du pays ${ }^{99}$ et dès les années 1590 , le roi recrute une armée de Cam et de «Malais» pour défendre Lovek contre les Siamois ${ }^{100}$. Vers 1598, Rām I ${ }^{\mathrm{er}}$, roi du Cambodge, envoie à Manille une ambassade dirigée par un Malais de Johor ${ }^{101}$. Cet individu originaire de l'Archipel (jvā) apparaît dans les chroniques royales du Cambodge sous le nom de Laksamana, qui renvoie au même terme bien connu dans le monde insulindien pour désigner un amiral. Son premier fait d'armes semble avoir été une expédition au Campā, probablement en 1596, où considérant qu'il a été mal reçu, il suscite une révolte, pille la capitale et rentre au Cambodge avec pièces d'artillerie et prisonniers. Le souverain lui confie alors de hautes charges et des terres qu'il administre ${ }^{102}$. En compagnie d'un Cam nommé Po Rat, il se proclame souverain sur son territoire et commence à soumettre d'autres provinces. En 1599, un conflit violent à Phnom Penh entre les Européens et Malais aboutit à l'assassinat de pratiquement tous les Européens par les troupes de Laksamana, qui font également disparaître le souverain Paramarājā V. Chassé du Cambodge par Paramarājā VI, il se réfugie au Campā où il meurt ${ }^{103}$.

On peut penser qu'un certain nombre de marchands musulmans ayant fui le Melaka portugais prennent la direction du port cam de Phan Rang, ou du port de Hoi An (Cua Dai Chiem) contrôlé par la cour des Nguyen à Hué, port qui devient au $\mathrm{XVI}^{\mathrm{e}}$ siècle un relais important entre le Japon, la Chine et l'Archipel. Hoi An est alors dominé par les marchands chinois, en particulier les Hokkien ${ }^{104}$, et le restera au moins jusqu'à la fin du XVII ${ }^{\mathrm{e}}$ siècle ${ }^{105}$.

98. Sanjay Subrahmanyam, "Manila, Melaka, Mylapore...: A Dominican Voyage through the Indies, ca. 1600", Archipel, 57, 1999 : 235-6 (223-242).

99. A. Reid, Southeast Asia in the Age of Commerce, 1450-1680, vol. II, New Haven/London, Yale University Press, 1993: 188. Ceux originaires de 1'Archipel sont souvent désignés comme Malais dans les sources. Or, des Javanais sont également présents (B.P. Groslier, Angkor et le Cambodge au XVI siècle d'après les sources portugaises et espagnoles, Paris, Presses Univ. de France, 1958 : note 4 p. 49).

100. D.G.E. Hall, A History of South-East Asia, Houndmills, New York, 1981 (1 ère éd., 1955): 281.

101. Subrahmanyam, $1999: 236$.

102. Mak Phoeun, 1995 : 64-66.

103. B.P. Groslier 1958 : 54-5; Mak Phoeun, 1995 : 89-91, 94.

104. Reid, 1993: 63-64; Charles Wheeler, "Re-Thinking the Sea in Vietnamese History: Littoral Society in the Integration of Thuận-Quảng, Seventeenth-Eighteenth Centuries", JSEAS, 37(1), 2006: 142 (123-153).

105. L.Y. Andaya, "Interactions with the Outside World and Adaptation in Southeast Asian Society, 1500-1800", in N. Tarling (ed.), The Cambridge History of Southeast Asia, Cambridge, Cambridge University Press, vol. I (part two), 1999 (1 1 ère éd., 1992): 6-7. 
Dans le récit de ses pérégrinations dans la région au cours des années 1530, Mendes Pinto rapporte que l'un des quatre bataillons de l'armée de Pasuruan, à Java Est, est commandé par un Cam ${ }^{106}$.

À Makassar, la première implantation permanente et identifiée comme telle d'une communauté malaise daterait des années 1550-60 selon la Chronique de Gowa. Sous l'autorité d'un capitaine nommé Anakoda Bonang, elle accueille alors des gens de Pahang, de Patani, du Campā, de Minangkabau et de Johor ${ }^{107}$. Un personnage portant le titre de Dato' (ou Datuk) Maharajalela est associé à cette communauté malaise de Makassar, dès le début des années 1630 selon la tradition locale ${ }^{108}$. Sa biographie est loin d'être claire. Une tradition lui attribue le nom de Andi (Faqih) Ali Datok Maharajalela, fils de Mustafa Datu Jambu, qui serait devenu souverain du Campā entre la fin du XVI ${ }^{\mathrm{e}}$ et le début du XVII ${ }^{\mathrm{e}}$ siècle. Installé un temps à Patani, Ali aurait d'abord pris le titre de Tok Koda Bonang avant de se rendre à Sulawesi. Ce titre n'est pas sans rappeler l'Anakoda Bonang cité plus haut. Il serait revenu ensuite à Patani, à l'époque de Raja Ungu, où il aurait prit le titre de Datu Sri Maharajalela ${ }^{109}$.

Au XVII ${ }^{e}$ siècle, le commerce de Patani avec le Cambodge est assuré au moins en partie, sinon en totalité, par des Chinois de Patani ${ }^{110}$. Le sultanat reçoit du bois d'aigle du Campā, du Cambodge et de la Cochinchine, des esclaves du Campā et du Cambodge, du bois de laque, des résines, du benjoin, des peaux de cervidés, de la cardamome, du coton, du sucre et des cuirs du Cambodge ${ }^{111}$. Au moins dès le tournant du XVII ${ }^{\mathrm{e}}$ siècle, il convient d'ajouter le riz, que Patani importe du Cambodge ${ }^{112}$. Il faut mentionner ici un autre petit détail concernant le Patani du XVII ${ }^{\mathrm{e}}$ siècle : lorsqu'en 1641, un bateau de la VOC commandé par Gerrit van Wuysthoff remonte le Mékong, il dispose à son bord d'un interprète malais de Patani ${ }^{113}$. Au Cambodge

106. Fernão Mendes Pinto, Pérégrination (trad. \& éd., R. Viale), Paris, La Différence, 1991 : 593.

107. A. Reid, "The Rise of Makassar", RIMA, 17, 1983: 137-8 (117-160).

108. W. Cummings, "The Melaka Malay Diaspora in Makassar (c. 1500-1669)", JMBRAS, LXXI(1), 1998: 109, 114-6 (107-122); H. Sutherland, "The Makassar Malays: Adaptation and Identity, c. 1660-1790", JSEAS, 32(3), 2001: 400 (397-421).

109. Mohd. Shaghir Abdullah, Syeikh Daud Bin Abdullah Al-Fatani. Ulama' dan Pengarang Terulung Asia Tenggara, Shah Alam, HIZBI, 1990: 9-10.

110. H. Terpstra, De factorij der Oostindische Compagnie te Patani, 's-Gravenhage, Martinus Nijhoff, VKI 1, 1938: 136.

111. Il est possible que ces échanges débutent dès l'apparition du sultanat à la fin du XVe siècle. Cf. D. Perret, «Patani dans les grands réseaux marchands du XVII e siècle», in D. Perret, A. Srisuchat \& S. Thanasuk (textes réunis par), Études sur l'histoire du sultanat de Patani, Paris, EFEO, Études Thématiques 14, 2004 : 250-253.

112. Reid, $1988: 21$.

113. M. Guérin, $2004: 34$. 
même, vers 1621, des «Malais», des Chinois et des Khmers sont envoyés pour chercher de l'or en amont de la Nam Noy ${ }^{114}$.

Durant les années 1630 au Cambodge, dans la capitale située à Oudong, les communautés étrangères sont représentées par plusieurs sabandar, y compris la communauté malaise. Lorsque Hagenaar, le premier envoyé de la VOC, se présente à la cour, sa missive est lue en malais, puis traduite en khmer ${ }^{115}$. En 1643/4, le roi Rāmādhipatī $\mathrm{I}^{\mathrm{er}}$, qui a pris le pouvoir par la force, se convertit à l'islam. Selon certaines versions des chroniques royales, cette conversion aurait fait suite à son mariage avec une musulmane, malaise ou cam selon les textes, et peut-être d'origine minangkabau. Il aurait également confié des charges importantes à des Malais. D'après d'autres versions des chroniques, c'est pris de remords après avoir fait assassiner son oncle pour accéder au trône, qu'il se convertit à l'islam, convaincu par les autorités religieuses malaises qu'il serait délivré de ses fautes par cette conversion. Face à une population hostile, il semble avoir cherché du soutien auprès des musulmans cam et malais vivant au Cambodge, prenant le nom de Sultan Ibrahim et s'entourant d'une garde de Javanais et Malais ${ }^{116}$. Il est chassé du pouvoir en 1659, à la suite d'une révolte de princes destinée à faire cesser la prépondérance des Malais au sein de la cour du Cambodge. Face à la disparition de leur protecteur et, par conséquent, de leurs privilèges et avantages, les Cam et Malais se révoltent dans la province de Thbaung Khmum. Finalement battus, les principaux dirigeants de cette révolte, y compris la reine, ainsi que nombre de partisans (plus de 700 familles) se réfugient au Siam ${ }^{117}$. Cependant, tous les Malais n'ont pas quitté le pays, puisqu'on sait que dès la fin des années 1660, il y a une communauté malaise à Phnom Penh, et qu'une décennie plus tard ils disposent toujours, ou ont retrouvé, une grande influence à la cour de Oudong. Le souverain de l'époque, Padumarājā II, est d'ailleurs assassiné par quatre Malais en 1673, sur ordre de la reine ${ }^{118}$.

Parmi les marchands malais fréquentant le Cambodge à l'époque, il y a des gens de Makassar qui appartiennent à un réseau s'étendant jusqu'à Manille ${ }^{119}$, où réside d'ailleurs une communauté marchande cam ${ }^{120}$. Lorsque Makassar passe sous contrôle de la VOC en 1669, un certain

114. Mak Phoeun, 1995 : 188.

115. Mak Phoeun, $1995: 233,236$.

116. Mak Phoeun, 1995 : 259-261.

117. Mak Phoeun, 1995 : note 225 p. 294, 307-8, 317.

118. Mak Phoeun, $1995: 317$.

119. Cummings, $1998: 110$; Sutherland, $2001: 399$.

120. Manguin $1979: 277$. 
nombre de marchands musulmans se réfugient au Cambodge, parmi d'autres places encore indépendantes en Asie du Sud-Est ${ }^{121}$.

À propos du Campā au tout début du XVII' siècle, l'amiral néerlandais Matelief, qui y fait escale en 1607, apprend que le dirigeant «hindou» est allié avec le sultan de Johor ${ }^{122}$. L'année précédente, l'Anglais Edmund Scott, qui réside alors à Banten, y note la présence d'une Cochinchinoise épouse d'un Chinois résident ${ }^{123}$. Toujours à propos de Banten, il faut signaler l'existence de relations commerciales avec le Campā dans la seconde moitié du siècle, puisque le sultan y envoie ses navires à la fin des années $1660^{124}$.

Il semble que dès le XVII ${ }^{\mathrm{e}}$ siècle, la côte orientale de la péninsule malaise, en particulier le sultanat de Kelantan, ait joué un rôle symbolique important pour les Cam musulmans, puisqu'ils associent Kelantan à La Mekke et y viennent en pèlerinage. Dès lors, au moins sur le plan religieux, les échanges se multiplient y compris pour lutter contre le Viêt Nam ${ }^{125}$. La contribution de Danny Wong à ce dossier revient sur cet aspect pour expliquer l'histoire récente de la communauté cam en Malaisie.

$\mathrm{Au}$ tout début du XVIII ${ }^{\mathrm{e}}$ siècle, on retrouve des gens de Makassar au cœur d'un événement sanglant au Viêt Nam. Il s'agit de la mutinerie de Pulo Condore, où les Anglais ouvrent une loge commerciale en 1702. Trois ans plus tard, mécontents du sort qui leur est fait, les soldats originaires de Makassar au service des Anglais, massacrent tous les Européens sauf deux, qui parviennent à s'enfuir à Johor ${ }^{126}$.

Â la fin du XVII ${ }^{\mathrm{e}}$ siècle, des marchands de 1'Archipel fréquentent Saïgon et My Tho dans le delta du Mekong, quelques années après que quelques milliers de Chinois, anciens partisans de Zheng Chenggong (Koxinga) défait

121. Sutherland, $2001: 398$.

122. "Relation du voiage de Corneille Matelief le jeune amiral hollandois aux Indes Orientales (1605-1608)», in Recueil des voiages qui ont servi à l'établissement et aux progrès de la Compagnie des Indes Orientales, Formée dans les Provinces Unies des Païsbas, Tome III, Amsterdam, Etienne Roger, 1705 : 500. Dès 1594 au moins, le roi du Campā envoie des troupes à Johor pour combattre les Portugais. Les bons rapports établis avec les Hollandais à cette occasion se confirment avec l'envoi par le dirigeant du Campā, en 1680, de deux ambassadeurs à Batavia et deux années plus tard, il envoie deux de ses navires commercer à Melaka (Manguin, $1979: 272,276,277$ ).

123. Edmund Scott, "An exact discourse of the Subtilities, Fashions, Pollicies, Religion, and Ceremonies of the East Indians, as well Chyneses as Javans, there abyding and dwelling", in W. Foster, ed., The Voyage of Sir Henry Middleton to the Moluccas (1604-1606), London, The Hakluyt Society, 1943: 127.

124. W.P. Coolhaas (éd.), Generale missiven van gouverneurs-generaal en raden aan Heren XVII der Verenigde Oostindische Compagnie. Deel III, 1655-1674, 's-Gravenhage, M. Nijhoff, 1968: 638.

125. Po Dharma, $2000: 185-6$.

126. D. Wong Tze-Ken, 2012. 
par le régime mandchou, se sont réfugiés à Hoi Han, d'où le souverain Nguyen les envoie dans la région du delta du Mékong. Ces Chinois y développent rapidement des marchés florissants ${ }^{127}$.

Une tradition locale veut que la mosquée de Kampung Laut, à Kelantan, près de Kota Bharu ait été édifiée par des Cam ${ }^{128}$. L'étude architecturale de cette mosquée a permis de suggérer une datation de construction au XVIII ${ }^{\mathrm{e}}$ siècle, ce qui s'accorderait bien avec cette tradition ${ }^{129}$.

Malgré ces siècles de contact, il faut cependant attendre la fin du XVIII siècle pour disposer des premiers comptes rendus de missions d'émissaires vietnamiens dans le monde insulindien, région désignée alors par l'expression Hạ châu. Ce sont ces sources relatives à des missions s'étalant jusqu'en 1846 que Claudine Salmon examine dans ce dossier.

Le $\mathrm{XIX}^{\mathrm{e}}$ siècle apporte des éléments sur les relations autres que le grand commerce. Ainsi, la Carita Bangka, un texte malais du milieu du XIX ${ }^{\mathrm{e}}$ siècle, mentionne la présence de travailleurs de Koci (Dai Viet) dans l'archipel, dont l'île de Bangka ${ }^{130}$.

Ce siècle voit surtout la mise en place de deux réseaux religieux parallèles entre la péninsule indochinoise et la péninsule malaise. Il s'agit d'une part du réseau stimulé par les écoles islamiques sur la côte Est, et d'autre part du réseau stimulé par le développement du Collège général des Missions Etrangères de Paris à Penang sur la côte Ouest.

Depuis le $\mathrm{XIX}^{\mathrm{e}}$ siècle au moins jusqu'à aujourd'hui, les écoles religieuses musulmanes (pondok et madrasah) de Pattani, Kelantan et Terengganu sont en effet régulièrement fréquentées par des étudiants cam et malais du Cambodge $^{131}$. Dans l'autre sens, probablement dès la fin du XVI $\mathrm{X}^{\mathrm{e}}$ siècle au moins, des ulémas malais fréquentent le Cambodge afin de propager la religion et de régler des questions religieuses ${ }^{132}$. Au XIX ${ }^{\mathrm{e}}$ siècle, sur la côte opposée, c'est un réseau missionnaire catholique qui est actif. Le Collège

127. Reid, $1993: 315$.

128. Guérin, 2004: 34.

129. Jacques Dumarçay, «La mosquée de Kampung Laut (Kelantan) : étude architecturale», Archipel, 44, 1992 : 115-122.

130. Malay Concordance Project. E.P. Wieringa, Carita Bangka, Het verhaal van Bangka, Leiden, Vakgroep Talen en Culturen van Zuidoost-Asië en Oceanië, Rijksuniversiteit te Leiden, 1990. Semaian 2. (1861): 106:13, 88:14, 88:9, 88:16, 88:23, 103:19, 88:11.

131. Ner, 1941: 152, 158-164, 167-8, 171; Lombard, 1987: 316; Hasan Madmarn, The Pondok \& Madrasah in Patani, Bangi, Penerbit Universiti Kebangsaan Malaysia, 1999: 123; Guérin 2004: 38. Le premier uléma documenté de Pattani est Shaykh Daud bin Abdullah bin Idris al-Fatani, dont la première œuvre connue a été achevée à La Mekke en 1809 (V. Matheson \& M.B. Hooker, "Jawi Literature in Patani: The Maintenance of an Islamic Tradition", JMBRAS, LXI(1), 1988: 20 (1-86)).

132. Voir la contribution de Danny Wong à ce dossier. Cf. également Ner, 1941 : 152; Guérin $2004: 39$. 
général des Missions Etrangères de Paris installé à Penang voit ainsi passer plus de 600 séminaristes envoyés du Viêt Nam, et 11 du Cambodge, entre 1830 et $1950^{133}$. L'un d'entre eux au moins fera une carrière brillante dans le domaine de la langue vietnamienne. Il s'agit de Truong Vinh Ky, appelé également Pétrus Ky (1837-1898), qui reçoit une formation au grand séminaire de Penang en 1852, avant de poursuivre ses études en France. Il est notamment l'auteur d'une Grammaire de la langue annamite (Saïgon, 1883), considérée comme la première véritable grammaire de la langue vietnamienne. Professeur à l'école des interprètes au collège des stagiaires de Saïgon, il sera également directeur du premier journal Gia dinh bao en vietnamien et membre du conseil secret de la cour d'Annam à Hué ${ }^{134}$.

\section{Les réseaux communistes}

L'un des aspects les moins connus des relations entre la péninsule indochinoise et le monde insulindien à l'époque contemporaine est relatif aux liens qu'entretenait le Parti Communiste de Malaya (MCP) avec, d'abord la Ligue pour l'indépendance du Viêt Nam (Viêt-Minh), ensuite le gouvernement de la République Démocratique du Viêt Nam. Créé en 1930, le MCP connaît des débuts difficiles, d'une part en raison de son interdiction par l'administration coloniale britannique, d'autre part en raison de dissensions internes qui vont pratiquement conduire à sa paralysie. Il faut l'arrivée d'un certain Lai Tek, un agent se présentant comme envoyé par l'Internationale Communiste (Comintern), pour remettre le parti sur pied. Ce Lay Tek était apparemment un communiste annamite d'abord arrêté par la police française avant d'être offert aux Services Spéciaux britanniques comme agent double pour infiltrer le MCP. Il devient Secrétaire Général du parti, le dirigeant pendant la Seconde guerre mondiale, au cours de laquelle sa branche armée, la Malayan Peoples' Anti-Japanese Army (MPAJA), combat parallèlement aux forces alliées. La MPAJA est dissoute après la guerre, tandis que le MCP tente d'opérer au grand jour.

À cette époque, le MCP fournit également de l'aide à la Ligue pour l'indépendance du Viêt Nam (Viêt-Minh) dans sa lutte contre les Français. En 1947, le Viêt-Minh lance en effet un appel à volontaires parmi les partis communistes des pays voisins afin de soutenir les efforts des communistes

133. Bernard Patary, Homo Apostolicus. La formation du clergé indigène au Collège général des Missions Étrangères de Paris, à Penang (Malaisie), 1808-1968 : institution et représentations, Université Lumière Lyon 2, Lyon, 2009 : tab. 3 - thèse en ligne consultée en novembre 2012.

134. Lê Thị Xuyến, Phạm Thị Quyên, Đỗ Quang Việt, Nguyễn Văn Bích, «Bref aperçu sur l'histoire de l'étude des parties du discours en vietnamien (1 ère période)», Histoire Epistémologie Language, 26(1), 2004 : 150 (137-158). Voir également l'article de Claudine Salmon dans ce volume sur un autre aspect du personnage. 
en Indochine. L'association des anciens camarades du MPAJA et ses antennes commencent à collecter des fonds pour porter assistance au ViêtMinh, en particulier en finançant ses «volontaires». Par ailleurs, un certain nombre d'anciens combattants du MPAJA, pressentant que le parti pourrait se lancer dans une guerre en Malaya, dans laquelle ils ne voient aucune chance de succès, se portent volontaires et sont envoyés en Indochine ${ }^{135}$. Deux ans après le début des actions viet-minh contre le régime colonial français, le MCP lance en juin 1948 une insurrection armée contre l'administration britannique en Malaya, insurrection qui va durer jusqu'en 1960, une période connue sous le nom d'Emergency.

Pour le MCP, la guerre en Indochine n'est probablement pas un souci majeur en ce qui concerne ses stratégies militaires en Malaya. Il convient de noter toutefois une exception, la Résolution de juin 1949, émise par le Bureau Politique Central, mettant l'accent sur l'idée que la lutte armée est la plus haute forme de lutte révolutionnaire. À cet égard, le politburo du MCP affirme :

«Il n'y a aucune aide internationale à attendre, si ce n'est sous forme de propagande et de soutien moral, jusqu'à ce que la MRLA [l'Armée de Libération Révolutionnaire de Malaya] elle-même parvienne à s'établir fermement par des gains significatifs de territoires dans le pays, comme les communistes l'ont fait en Chine et en Indo-Chine. Ainsi, les camarades ne peuvent compter que sur leurs propres efforts sur une longue période pour atteindre cette étape, sans espoir d'une aide étrangère pour sauver actuellement la révolution en Malaya.» ${ }^{136}$

L'Indochine est toutefois importante pour les leaders du MCP comme lien avec le Parti Communiste en Chine. Des témoignages de dirigeants du MCP, y compris Chin Peng, révèlent comment ils se rendaient en Chine méridionale par voie terrestre à travers le nord de la Malaya, la Thaillande, le Laos et le nord du Viêt Nam. C'est avec le repli du MCP sur la frontière Malaya-Thaïlande, que des relations plus étroites se tissent avec l'Indochine. Entre 1954 et 1963, l'Indochine continue de servir de voie principale pour les cadres du MCP se rendant en Chine. Au début des années 1960, alors que le conflit s'intensifie entre le Viêt-Minh et le gouvernement de Ngo Dinh Diem basé à Saïgon, le MCP se tourne vers Hanoi pour chercher son inspiration. Une mission du MCP s'y installe en 1961, précisément rue Tran Hung Dao. Elle devient un lieu de transit très utile aux dirigeants du MCP en route pour la Chine ${ }^{137}$.

135. C.C. Too, "Notes on the History of the Communist Party of Malaya", University of Malaya Library, 1990: 67-68.

136. "The General History of the Party" in C.C. Too Papers: 105.

137. Aloysius Chin, The Communist Party of Malaya: the Inside Story, Kuala Lumpur, Vinpress, 1994: 183-184. 
Bien qu'il n'y ait pas de preuve de soutien militaire direct vietnamien (Parti Lao Dong/Parti des Travailleurs) au MCP, les tactiques militaires vietminh et plus tard celles du Front de Libération Nationale sont grandement appréciées par les cadres du MCP. Suite à 1'installation de sa mission à Hanoi, certains d'entre eux sont invités par le Comité Central du Parti Lao Dong à participer à ses cours de formation. Parmi les tout premiers cours mis en place, il y a notamment celui de 1961 destiné spécialement aux cadres du MCP, intitulé «l'expérience révolutionnaire vietnamienne» ${ }^{138}$. Avec l'ouverture des écoles du MCP sur la frontière Thai-Malaya cette même année 1961, les stratégies et tactiques militaires viet-minh/viet-cong font partie intégrante du module de formation. C'est le cas notamment à l'école numéro cinq ${ }^{139}$.

En Malaya, il faut attendre 1953 pour que l'attention du public se porte sur les événements qui se déroulent alors en Indochine. L'événement marquant est l'incursion des troupes viet-minh en territoire laotien en marsavril 1953, incursion qui menace Luang Prabang, la capitale royale du pays ${ }^{140}$. Il est rapporté dans la presse en Malaya comme pouvant avoir des implications à long terme en Malaya même, la crainte étant qu'il pourrait susciter une réaction en chaîne dans des pays d'Asie du Sud-Est confrontés à des rébellions armées dirigées par des communistes. En fait les progrès du Viêt-Minh eurent une influence directe sur le moral des membres du MCP. Ainsi, alors que le Viêt-Minh enregistre des gains territoriaux significatifs au Viêt Nam et au Laos, en Malaya les autorités constatent une baisse significative du taux de défection au MCP, dans le cadre des plans d'amnistie offerts par le gouvernement ${ }^{141}$.

\section{Relations diplomatiques avant 1975}

À l'époque contemporaine, les relations diplomatiques entre la péninsule indochinoise et le monde insulindien débutent avec l'établissement de relations officielles entre 1'Indonésie et la République Démocratique du Viêt Nam indépendant en 1954 (à l'époque du régime communiste dirigé par Hô Chi Minh). L'année suivante, l'Indonésie accueille le sommet du Mouvement des Non Alignés à Bandung, sommet auquel assiste Hô Chi Minh. C'est aussi en 1954 qu'est fondée l'Organisation du Traité de l'Asie du Sud-Est (SEATO), une initiative des États-Unis et des nations anti-

\section{Ibid.: 184 .}

139. Ibid.: 98-99. Durant les années 1970, le Viêt Nam est l'une des régions où le MCP envoie ses cadres pour des formation en tactique militaire.

140. Malay Mail, 25 April 1953.

141. Télégramme de Sir Gerald Templer à Secretary of State for the Colonies, 18 June 1953, CO1022/49 [Colonial Office: South East Asia Department, Original Correspondence]. 
communistes pour enrayer l'avancée de l'influence communiste et de possibles conflits militaires en Asie du Sud-Est. Si la Malaya elle-même n'y participe pas, son colonisateur en est membre et la Grande-Bretagne va fournir une assistance à la SEATO.

Entre l'indépendance de la Malaya en 1957 et la chute de Saïgon en 1975, la Malaisie maintient des relations diplomatiques avec la République du Viêt Nam (Sud Viêt Nam) et ne reconnaît la République Démocratique du Viêt Nam qu'en 1973, à la suite de l'Accord de paix de Paris. Cette prise de position était basée sur la longue et amère expérience de l'insurrection communiste armée. Même si la période de l'Emergency est considérée comme terminée en 1960, la rébellion armée du MCP se poursuit en fait jusqu'en 1989. C'est ce contexte de fort positionnement anti-communiste qui va déterminer les relations bilatérales avec les deux Viêt Nam. Après l'indépendance, la Malaya envoie des surplus d'armes et de véhicules blindés au régime de Saïgon ${ }^{142}$. Mais contrairement à ses voisins, la Thaïlande et les Philippines, la Malaisie n'enverra jamais de troupes tout en restant un fervent soutien du Sud Viêt Nam, y compris dans la plupart des forums internationaux, notamment les Nations-Unies. Elle mettra par ailleurs l'île de Penang comme lieu de repos et de distraction à disposition des troupes américaines servant en Indochine ${ }^{143}$.

Durant la Guerre du Viêt Nam, les experts anti-insurrection américains et sud vietnamiens voient la Malaya comme un exemple de succès dans la lutte anti-communiste. Sir Robert Thompson, un officier supérieur britannique en poste en Malaya est invité comme conseiller pour la mise en place de mesures destinées à éliminer l'ennemi communiste au Viêt Nam. Parmi les mesures adoptées figure l'établissement de «hameaux stratégiques », suivant en cela le Briggs Resettlement Plan qui, en Malaya, prévoyait le déplacement de villageois jugés vulnérables à la menace communiste dans des hameaux où une protection serait assurée. Mais contrairement à la Malaya où ce plan est mis en place de façon stricte, le manque de volonté politique et les carences de l'administration du régime de Saïgon entraînent son échec, malgré sa réintroduction par des régimes successifs ${ }^{144}$.

Parallèlement, les contacts religieux se poursuivent entre la péninsule indochinoise et la Malaisie dans les années 1960. Des candidats venus du

142. D. Wong Tze-Ken, Vietnam-Malaysia Relations during the Cold War, 1945-1990, Kuala Lumpur, University of Malaya Press, 1995: 48.

143. Khaw Guat Hoon, Malaysian Policies on Southeast Asia: The Search for Security, PhD Dissertation, Université de Genève, Genève, 1976: 125-133.

144. Voir Milton E. Osborne, Strategic Hamlets in South Viet-Nam: A Study and Comparison, Data Paper No. 55, Southeast Asian Studies Program, Department of Asian Studies, New York, Ithaca, Cornell University, 1965; voir aussi Robert Thompson, Defeating Communist Insurgency, The Lessons of Malaya and Vietnam, New York, Praeger Publishers, 1966: 121-140. 
Viêt Nam et du Cambodge participent régulièrement aux concours internationaux annuels de récitations coraniques organisés par le gouvernement malaisien. Dans l'autre sens, des lettrés religieux de Malaisie continuent d'effectuer des visites prolongées dans les deux pays, afin de fournir une instruction religieuse aussi bien aux Malais qu'aux Cam. Des publications à caractère religieux sont également importées de Malaisie et de Singapour ${ }^{145}$.

C'est au moment où l'Indonésie lance sa campagne de Confrontation contre la nouvelle Fédération de Malaysia en 1963-66, que s’interrompt le soutien direct de la Malaisie au Sud Viêt Nam. Ceci afin de permettre à la Malaisie de faire face à la menace indonésienne. Les deux Viêt Nam vont réagir différemment. Bien que le soutien de la Malaya (et plus tard de la Malaisie) soit sans commune mesure avec celui offert par ses voisins, ce retrait provoque une réaction des dirigeants du Sud Viêt Nam réclamant la poursuite de cette aide. De son côté, la République Démocratique du Viêt Nam (Nord Viêt Nam) appuie fermement 1'Indonésie dans sa volonté d'écraser la Malaisie en condamnant cette dernière comme le fruit d'un complot néo-colonialiste britannique. Hô Chi Minh déclare son «soutien sans réserve» à Soekarno contre la Malaisie, "une création des Impérialistes » ${ }^{146}$. Toutefois, après la chute de Soekarno et la fin de la Confrontation en 1966, le pouvoir en place en Indonésie commence à prendre ses distances vis-à-vis de Hanoi.

La création en 1967 de l'ASEAN par cinq Etats non communistes d'Asie du Sud-Est (Thaïlande, Malaisie, Singapour, Indonésie et Philippines) va contribuer aux efforts anti-communistes à travers le développement économique et social. Si le régime de Saïgon accueille favorablement cette initiative, elle est par contre condamnée par le gouvernement de la République Démocratique.

Durant les années 1970, les changements dans l'environnement international culminent avec la signature de l'Accord de Paix de Paris en 1973. Le cessez-le-feu au Viêt Nam s'accompagne d'un relâchement au moins temporaire des tensions régionales, se traduisant notamment par 1'ouverture de relations diplomatiques entre Kuala Lumpur et Hanoi ${ }^{147}$. L'interruption des hostilités sera courte puisque le Nord Viêt Nam défait le Sud Viêt Nam en avril 1975, ce qui conduit à la réunification du pays l'année

145. Socialist Republic of Vietnam, Government Committee for Religious Affairs, Religion and Policies Regarding Religions in Vietnam, Hanoi, Socialist Republic of Vietnam Government Committee for Religious Affairs, 2006: 25-26.

146. Hô Chi Minh cité dans Arnold C. Brackman, Southeast Asia's Second Front: The Power Struggle in the Malay Archipelago, New York, Frederick A. Praeger, 1966: 284. Voir également Nhan Dan, 27/12/1962.

147. Foreign Affairs Malaysia, 6(1), 1973: 92. 
suivante. Néanmoins, cette dernière ouvre la voie à la reconnaissance de la République Socialiste du Viêt Nam par les Etats de l'ASEAN, y compris la Malaisie et l'Indonésie. De même, Malaisie et Indonésie reconnaîtront les régimes communistes qui prennent le pouvoir au Cambodge et au Laos.

\section{Des boat people aux pembantu rumah cambodgiennes}

Les années qui suivent la réunification ne sont pas particulièrement marquées par des relations pacifiques entre le Viêt Nam et le monde insulindien. Après une période initiale de coexistence paisible, où les gouvernements de deux régions cherchent à s'adapter au nouveau contexte international, l'atmosphère de confrontation renaît. Trois problèmes majeurs vont dominer cette période dans les relations qui nous intéressent ici.

Le premier est relatif aux réfugiés, qui commence par un exode de populations de la péninsule indochinoise refusant de vivre dans les nouveaux régimes communistes. Nous ne reprendrons pas ici l'exposé détaillé des causes et du contexte de ces migrations, qui ont été suffisamment étudiés ailleurs. Rappelons toutefois que l'augmentation dans des proportions alarmantes du nombre de ces réfugiés va provoquer une double crise majeure pour la Malaisie : avec l'Indonésie d'un côté et avec le Viêt Nam de l'autre. Hanoi était en effet suspecté d'encourager l'exode massif de gens jugés non désirables par le pays. Ce problème avec le Viêt Nam ne sera finalement résolu qu'en 1992. Dans ce dossier, c'est tout particulièrement la situation des réfugiés cam en Malaisie qui est abordée, d'une part d'un point de vue global par Danny Wong Tze Ken, d'autre part illustré à travers un cas d'intégration dans la société malaisienne par Siti Nor Awang.

Le second problème est la crise au Cambodge. En décembre 1978, l'armée vietnamienne envahit le Cambodge, alors dirigé par le régime communiste des Khmers Rouges, en invoquant un effort humanitaire pour mettre fin aux massacres en masse perpétrés par ces derniers. La communauté internationale condamne toutefois le Viêt Nam pour cette décision considérée comme un acte d'agression envers un pays tiers. Plus important encore étaient les craintes suscitées par cette invasion qui pouvait replonger la région dans l'instabilité. La décision vietnamienne était en effet perçue comme l'événement précurseur de la réalisation de la théorie des dominos, théorie largement exposée dans les années 1950 et au début des années 1960, selon laquelle la chute des pays de l'Indochine déboucherait sur la domination totale du communisme dans l'ensemble de l'Asie du SudEst ${ }^{148}$. Des pays comme la Malaisie et l'Indonésie apporteront leur soutien

148. Cette théorie a été popularisée lors d'une conférence de presse du président américain Dwight D. Eisenhower en avril 1954. 
aux forces anti-vietnamiennes au Cambodge, rendant l'atmosphère politique d'autant plus tendue.

Le troisième problème concerne les disputes territoriales en mer de Chine méridionale, en particulier la question de l'archipel de Spratly. La perspective de fonds sous-marins au riche potentiel en ressources incite le Viêt Nam à revendiquer des droits face à la Chine et au reste de l'Asie du Sud-Est. À deux reprises au moins depuis 1976, la marine vietnamienne s'est ouvertement confrontée à la marine chinoise à ce propos, menaçant à la fois la sécurité des routes maritimes internationales passant dans la région et la stabilité politique régionale. L'archipel de Spratly reste aujourd'hui un point de discorde, moins entre le Viêt Nam et la Malaisie ou l'Indonésie qu'entre le Viêt Nam et les Philippines et la Chine. Comme le Viêt Nam, cette dernière revendique sa souveraineté sur la totalité de la mer de Chine méridionale, ce qui inclut non seulement 1'archipel de Spratly, mais également d'autres îles.

On peut dire qu'entre 1978 et 1996, les contacts entre la péninsule indochinoise et le monde insulindien sont au plus bas. Le Viêt Nam est alors marginalisé internationalement, décalé et en retard. Virtuellement sans accès aux fonds de la Banque Mondiale et du Fonds Monétaire International pour sa reconstruction, le pays se tourne vers l'Union Soviétique qui connaît alors d'importantes difficultés économiques. Cette impasse sur le plan international conduit en 1986 au tournant majeur bien connu dans sa politique économique. Cette nouvelle orientation débouche sur des rapprochements avec ses voisins, d'autant plus que le Viêt Nam réintègre progressivement la scène internationale suite au retrait de ses troupes du Cambodge en 1989, et que la question des réfugiés est résolue trois ans plus tard.

$\mathrm{Au}$ Cambodge, le retrait de l'armée vietnamienne laisse un vide dans un pays ravagé par la guerre. Avec une coalition au pouvoir incapable de mettre en place le futur politique du pays, les Nations-Unies prennent en charge son administration à travers 1'United Nations Transitional Authority in Cambodia (UNTAC) en 1992-1993. Des armées de plusieurs pays sont sollicitées et la Malaisie contribue en envoyant un bataillon qui aura une tâche d'observation et de maintien de la paix. Les troupes malaisiennes, issues essentiellement du First Royal Ranger Battalion ${ }^{149}$, vont également fournir une aide aux populations et contribuer à remettre sur pied la communauté musulmane.

En 1994, les Etats-Unis lèvent leur embargo économique sur le Viêt Nam, qui rejoint l'ASEAN l'année suivante. Le volume des échanges commerciaux commence alors à croître avec ses voisins sud-est asiatiques, de même que l'investissement étranger direct. Un développement similaire

149. New Straits Times, 30/03/2012. 
se produit au Cambodge. La Malaisie et Singapour constituent à cette époque les principaux investisseurs de la région dans les deux pays, pour qui ils deviennent également d'importants partenaires commerciaux ${ }^{150}$. Cette évolution s'accompagne logiquement de l'installation ou de déplacements fréquents d'hommes d'affaires, surtout chinois, ainsi que de techniciens et d'employés hautement qualifiés. Ces déplacements sont à leur tour facilités par la multiplication des liaisons aériennes entre les deux ensembles, surtout depuis une dizaine d'années ${ }^{151}$.

Le phénomène de migrations économiques s'observe également à grande échelle entre la péninsule indochinoise et la Malaisie depuis les années 2000.

Après avoir renvoyé en Indonésie plusieurs dizaines de milliers d'immigrants clandestins à la fin des années 1990, la Malaisie se tourne alors vers d'autres pays pour satisfaire ses besoins en main-d'œuvre dans divers secteurs. Elle commence à accueillir un nombre significatif de travailleurs migrants vietnamiens à partir de 2002, avec près de 20000 individus pour cette seule année, dont 1500 femmes ${ }^{152}$. Elle en aurait accueilli officiellement plus de 190000 en dix ans (avec un pic en 2006 - près de 38000 -, et une moyenne de 10000 annuellement en 2010 et 2011), dont plus de $70 \%$ dans le secteur manufacturier, le reste se répartissant entre la construction, l'agriculture, les services et le personnel domestique ${ }^{153}$. Au milieu des années 2000, le nombre de travailleurs originaires du Viêt Nam présents en Malaisie dépassait 100000 personnes, dont un quart de femmes ${ }^{154}$.

150. Ainsi, le groupe bancaire malaisien Maybank ouvre sa première succursale à Phnom Penh en 1994, opération suivie par l'ouverture de sa première succursale à Hanoi deux années plus tard (Maybank Annual Report 2010;

http://www.maybank.com/files/Maybank\%20Annual\%20Report\%202010.pdf) (consulté en nov. 2012).

151. Aujourd'hui, en tenant compte uniquement des compagnies aériennes de Malaisie et de Singapour, plus de 25 vols directs relient quotidiennement Kuala Lumpur et Singapour à Ho Chi Min City, Hanoi, Phnom Penh, Da Nang et Siem Reap, la ligne Kuala Lumpur-Ho Chi Min City, inaugurée en 1990 par Malaysian Airlines, étant la mieux desservie avec sept vols quotidiens dans les deux sens.

152. Labour Migration: Trends, challenges and policy responses in countries of origin. International Organization for Migration, Genève, 2003

http://www.iom.org.bd/publications/12.pdf (consulté en nov. 2012).

153. Vietnam Chamber of Commerce and Industry.

http://www.vccinews.com/news_detail.asp?news_id=23003, 21/04/2011 (consulté en nov. 2012).

154. Labour migration from Viet Nam: issues of policy and practice/ Dang Nguyen Anh; International Labour Office; ILO Regional Office for Asia and the Pacific, Asian Regional Programme on Governance of Labour Migration, Bangkok, 2008.

http:/www.ilo.org/wcmsp5/groups/public/—asia/—ro-bangkok/documents/publication/wcms_099172.pdf (consulté en nov. 2012). 
Une seconde composante dans les migrations économiques de la péninsule indochinoise vers la Malaisie concerne le personnel de maison d'origine cambodgienne. D'ampleur bien inférieure au précédent, ce flux a débuté en 2008, devenant significatif dès l'année suivante. En effet, suite à la multiplication de cas de meurtres et mauvais traitements à l'encontre de personnels de maison d'origine indonésienne en Malaisie ${ }^{155}$, le gouvernement indonésien met en place, en juin 2009, un moratoire sur l'envoi de personnel de maison en attendant que le gouvernement malaisien accorde des garanties sur les rémunérations et la protection des employées. Malgré la signature d'un accord entre les deux gouvernements en mai 2011, l'envoi de personnel de maison indonésien par l'intermédiaire d'agences agréées n'a toujours pas repris début 2013. Pour faire face à la pénurie de personnel de maison, les agences de recrutement malaisiennes se tournent notamment vers le Cambodge. Début 2011, la Malaisie comptait ainsi quelque 40000 travailleurs migrants d'origine cambodgienne, dont 25000 dans le secteur domestique ${ }^{156}$. La multiplication des cas de mauvais traitements conduit toutefois le gouvernement cambodgien, en octobre 2011, à interdire lui aussi à ses citoyens de travailler comme employées de maison en Malaisie ${ }^{157}$.

Le retour de la paix au Viêt Nam et au Cambodge voit également la reprise et le développement, surtout au Cambodge, des activités de missionnaires musulmans venus de Malaisie, d'organisations gouvernementales et non gouvernementales islamiques malaisiennes, ainsi que le financement de la construction de mosquées, surau, écoles islamiques et orphelinats avec l'aide de capitaux malaisiens et indonésiens ${ }^{158}$. Par

155. Avant 2009, le nombre total des employées de maison d'origines étrangères (surtout indonésienne et philippine) en Malaisie est estimé à 300000 individus (The Star, Kuala Lumpur, 29/02/2008, consulté en novembre 2012).

156. Human Rights Watch, 2011 report, http://www.hrw.org/sites/default/files/reports /cambodia1111webwcover.pdf (consulté en novembre 2012).

157. The Sundaily (23/11/2011); The Star (02/02/2013).

158. À titre d'exemples :

The Cambodia Daily, September 13, 2003;

http://www.camnet.com.kh/cambodia.daily/selected_features/putting_down.htm;

Regional Islamic Da'wah Council of Southeast Asia and the Pacific,

http://www.riseap.org/2010/07/29/visit-to-phnom-penh-cambodia/ (consulté en nov. 2012);

Report on the Fieldwork conducted in Malaysia and Cambodia on Muslim NGOs in Cambodia from 5 March to 17 March 2010 (27/04/2010), Organization for Islamic Area Studies, Waseda University;

http://www.kikou.waseda.ac.jp/ias/en/research/nihu2.php?id=77); http://khmernz.blogspot.com/2009/04/malaysia-to-help-cambodian-muslims.html;

Muslims In Cambodia Hoping For Assistance From Malaysian Government, NGOs (http://www.camboguide.com/cambodia-news-latest/muslims-in-cambodia-hoping-forassistance-from-malaysian-government-ngos); 
ailleurs, la formation des étudiants cam venant en Malaisie n'est plus limitée aux pondok et madrasah de la côte Est de la péninsule, puisque depuis la fin des années 1990 au moins, certains fréquentent des universités islamiques ${ }^{159}$.

Cette ouverture concerne aussi le champ matrimonial. En Malaisie, sur la totalité des mariages conclus entre 2009 et octobre 2011, 30\% des épouses étrangères sont vietnamiennes. Cette situation concerne en moyenne 2500 mariages avec des Chinois chaque année. Peu exigeantes, semble-t-il, sur l'aspect physique et la situation personnelle du conjoint, elles ont également souvent l'avantage d'apprendre rapidement le mandarin ${ }^{160}$.

\section{Conclusion}

Ce rapide panorama chronologique a permis de mettre en lumière, pour les périodes les plus anciennes, l'existence d'espaces et de réseaux caractérisés par des traditions et des pratiques communes dans les deux ensembles, traditions et pratiques qui suggèrent l'existence de contacts. À partir du milieu du second millénaire de notre ère et jusqu'au milieu du XIX ${ }^{\mathrm{e}}$ siècle, ces relations se précisent, ce qui permet d'en évaluer toute la diversité, malgré le caractère fragmentaire et quelquefois peu fiable, des sources. Pour la période qui débute au milieu du XIX ${ }^{\mathrm{e}}$ siècle, la multiplication et la précision de certaines sources permet d'envisager d'étudier ces relations à travers des parcours individuels notamment. Un pic dans ces contacts est atteint entre la fin des années 1970 et la fin des années 1980 avec l'afflux de réfugiés dans l'Archipel, réplique à grande échelle de l'épisode des réfugiés cam dans 1'Archipel après la prise de Vijaya par les Vietnamiens en 1471. Le second pic a débuté il y a dix ans avec, entre autres, des migrations économiques dans les deux sens, mais encore une fois surtout dans le sens péninsule indochinoise-monde insulindien, migrations facilitées par l'accroissement rapide des transports aériens. À l'inverse, le monde insulindien, en particulier la Malaisie, accroît ses actions en faveur des communautés musulmanes de la péninsule indochinoise.

Tout en reconnaissant le caractère très spéculatif de cette approche en raison de sources fragmentaires, on peut néanmoins tenter d'examiner ces relations sur la longue durée en termes de flux et de centres de gravité. Si l'on examine le domaine religieux par exemple, pour lequel nous disposons

PKPU (Persatuan Kakitangan Perkhidmatan Ugama) Teruskan Misi Dakwah \& Pendidikan di Kemboja Vietnam - 8/11/2012 (http://www.islam.gov.my/pkpu-teruskan-misi-dakwahpendidikan-di-kemboja-vietnam) (consultés en nov. 2012); "Pejabat Mufti Kemboja terima van", Berita Harian, 31/12/2012.

159. Regional Islamic Da'wah Council of Southeast Asia and the Pacific, http://www.riseap.org/2010/07/29/visit-to-phnom-penh-cambodia/ (consulté en nov. 2012).

160. The Star 22/11/2011. 
de quelques jalons sur un millénaire et demi, il semble qu'au milieu du premier millénaire de notre ère, le Funan centré sur le delta du Mékong a pu représenter un centre de gravité important pour le monde insulindien indianisé. À l'époque de Majapahit, les Javanais incluent le Campā parmi les régions ayant fourni des acteurs importants de l'islamisation de l'île. Un autre centre de gravité religieux apparaît dans les relations au $\mathrm{XV}^{\mathrm{e}}$ siècle, c'est Melaka. Depuis le XVII ${ }^{e}$ siècle, et on peut dire jusqu'à une époque récente, c'est la côte orientale de la péninsule malaise, en particulier Kelantan mais aussi Patani, qui prend le relais comme centre de gravité pour les communautés islamiques de la péninsule indochinoise. En sens inverse, un flux de missionnaires et volontaires relie jusqu'à aujourd'hui la péninsule malaise et le Cambodge en particulier. Parallèlement, Penang sur la côte ouest devient centre de gravité pour les catholiques de la péninsule indochinoise, surtout du Viêt Nam, entre le milieu du XIX ${ }^{\mathrm{e}}$ et le milieu du $\mathrm{XX}^{\mathrm{e}}$ siècle.

On pourrait aussi aborder l'histoire de ces relations de façon thématique. Ainsi, des travaux ont déjà porté sur la littérature malaise ou acihaise et

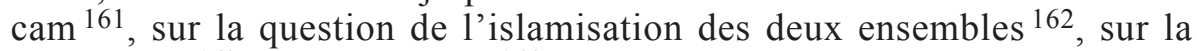
linguistique ${ }^{163}$ et la lexicologie ${ }^{164}$. Bien d'autres aspects sont envisageables, qu'il s'agisse des communautés ou individus médiateurs entre les deux régions, des rouages économiques transrégionaux, des réseaux religieux, des frontières, de l'imaginaire, de la circulation des techniques, des idées, des arts, des symboles, de divers produits, etc...

À titre tout à fait exploratoire, on notera par exemple que la littérature malaise traditionnelle ne semble pas avoir de concept pour désigner l'Asie du Sud-Est continentale ou la péninsule indochinoise ${ }^{165}$. On y trouve l'expression «benua China», mais lorsque dans un texte le passage concerne un déplacement vers la Chine, généralement rien n'est dit sur 1'Asie du Sud-

161. Cowan, 1933; G. Moussay, Akayet Deva Mano, Paris, EPHE, 1975 ; Nara Vija, Une épopée classique cam : Inra Pātrā, Paris, EPHE, 1975; Chambert-Loir, 1988 et 1994; Akayet Deva Mano: Versi Cam dari Hikayat Dewa Mandu, Kuala Lumpur, Kementerian Kebudayaan, Kesenian dan Pelancongan Malaysia/EFEO, 1989; Po Dharma; Moussay, G. \& Abdul Karim (eds.), Akayet Inra Patra (Hikayat Inra Patra - Epopée Inra Patra), Kuala Lumpur, Perpustakaan Negara Malaysia/EFEO, 1997; Po Dharma; Moussay, G. \& Abdul Karim (eds.), Akayet Dewa Mano (Hikayat Dewa Mano - Epopée Dewa Mano), Kuala Lumpur, Perpustakaan Negara Malaysia/EFEO, 1998; Po Dharma, G. Moussay, Abdul Karim (eds.) 2000. Un rapprochement a aussi été fait entre les littératures javanaise et khmère avec les Histoires de Panji (Lombard, 1987 : 317). Voir également G.E. Marrison, "The Chams and their Literature", JMBRAS, 58(2), 1985: 45-70.

162. Manguin, 1979; Lombard, 1987; Nakamura, 2000.

163. Cowan, 1948; Thurgood, 1999; Po Dharma, 1999.

164. Po Dharma, 1999.

165. Cette observation et les suivantes ont été effectuées par recherche systématique dans le corpus du Malay Concordance Project. 
Est continentale. Nous avons mentionné plus haut le toponyme Cempa/Campa, que ce soit dans les inscriptions en vieux-javanais ou dans plusieurs textes malais, les toponymes Kamboja et Yawana en vieuxjavanais, le toponyme Bal dans la littérature malaise pour désigner l'une des capitales du Campā, Kuci ou Koci pour désigner le Dai Viet, Kemboja/Kamboja dans la littérature malaise pour désigner le Cambodge. Il convient de mentionner ici le cheminement linguistique intéressant d'un toponyme que la littérature malaise situe «dekat Kemboja» (au Cambodge). Il s'agit de Pantai Emas (litt. Côte d'Or) mentionné dans trois textes malais ${ }^{166}$, et qui désigne en fait Banteay Meas (litt. la «Citadelle dorée» ou «la Citadelle d'or» en khmer), fondé par un Ming réfugié à la fin du XVII ${ }^{\mathrm{e}}$ siècle, un site présentement au Cambodge. Sa destruction par les armées siamoises va obliger son fondateur à se replier à l'embouchure de la rivière du même nom (sting Banteay Meas) sur un lieu connu aujourd'hui sous le nom de Ha Tiên, en territoire vietnamien cette fois ${ }^{167}$. À l'inverse, dans des lexiques Cam/Malais du XVII ${ }^{e}$ siècle, on trouve 1'expression «orang Benua» pour désigner les indigènes de la péninsule malaise, et les termes layu, liya, liyu, aliyu, pour désigner les Malais, le pays des Malais et la langue malaise ${ }^{168}$.

166. Malay Concordance Project. Muhammad Yusoff Hashim (ed.), Hikayat Siak dirawikan oleh Tengku Said, Kuala Lumpur, Dewan Bahasa dan Pustaka, 1992 (1855 -MCP). MCP: 476, 477; Virginia Matheson Hooker, Tuhfat al-Nafis: Sejarah Melayu-Islam, Kuala Lumpur, Dewan Bahasa dan Pustaka, 1991. (1866, MCP): MCP 168:7, 168:8; 168:9, 168:13, 170:3; Annabel Teh Gallop (with E. Ulrich Kratz), The Legacy of the Malay Letter: Warisan Warkah Melayu, London, British Library for the National Archives of Malaysia, 1994: 221, doc. $70(1820)$.

167. Les auteurs remercient Grégory Mikaélian et Claudine Salmon pour les informations concernant Banteay Meas, ainsi que pour les compléments bibliographiques apportés à la liste jointe à ce texte.

168. Po Dharma, $1999: 355,361$. 


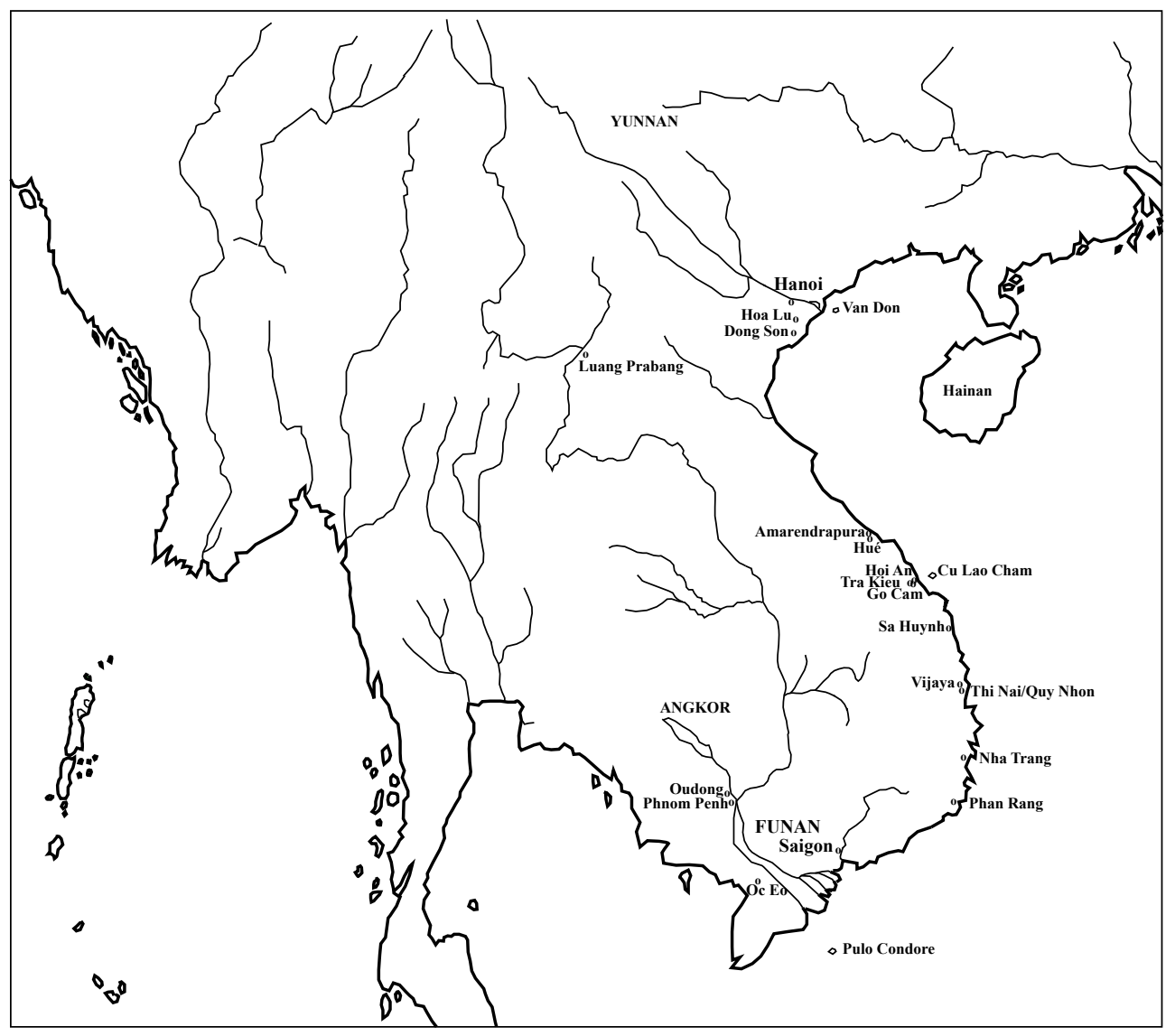

Péninsule indochinoise : principaux toponymes cités 
Daniel Perret \& Danny Wong Tze Ken

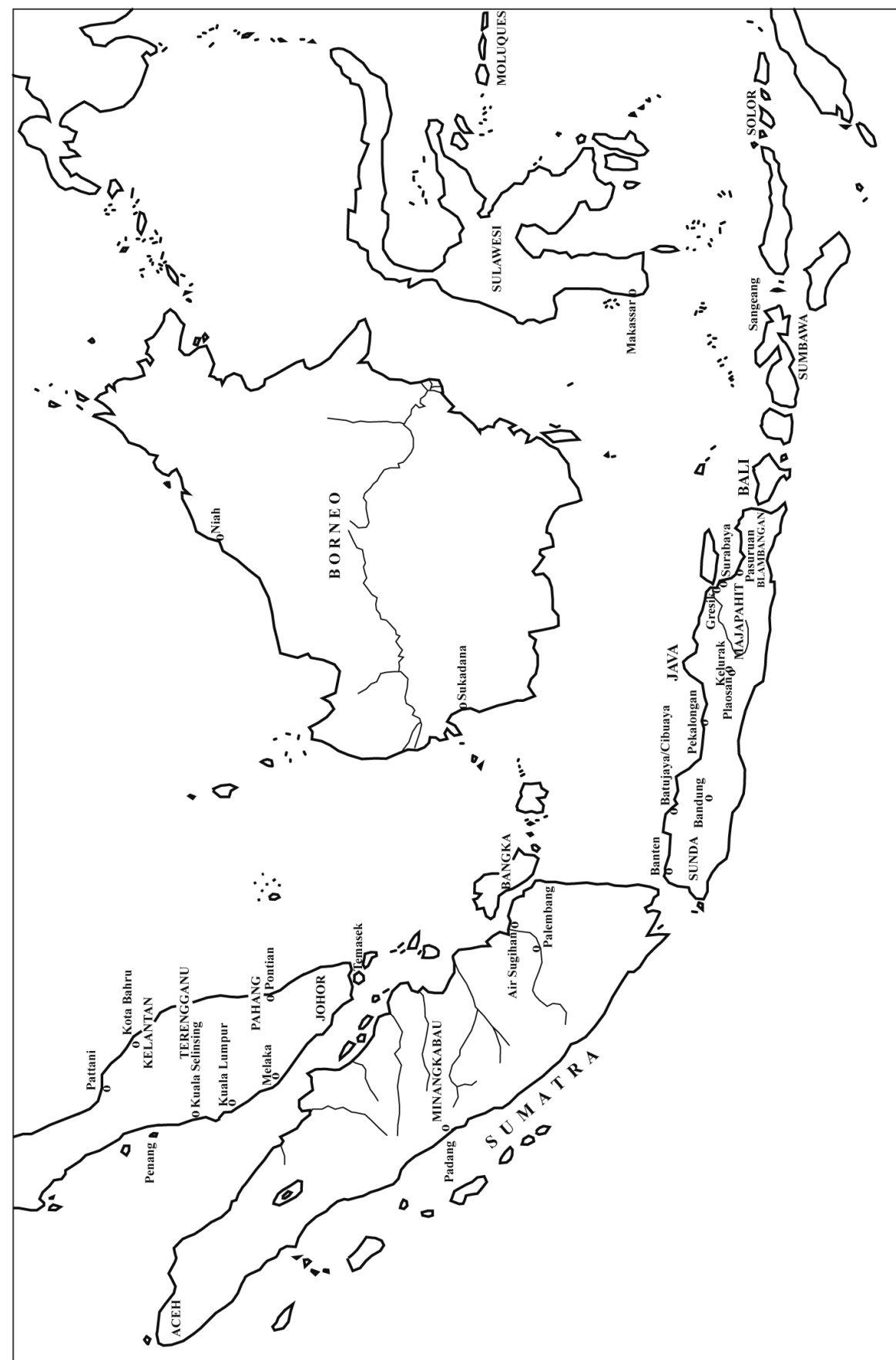

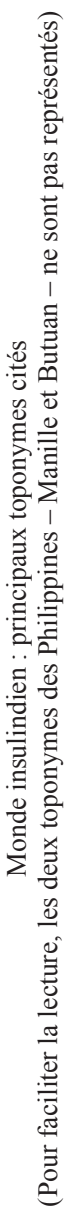

